\title{
Platelet-derived HMCB1 is a critical mediator of thrombosis
}

\author{
Sebastian Vogel, ${ }^{1}$ Rebecca Bodenstein, ${ }^{1}$ Qiwei Chen, ${ }^{2}$ Susanne Feil, ${ }^{3}$ Robert Feil, ${ }^{3}$ Johannes Rheinlaender, ${ }^{4}$ Tilman E. Schäffer, ${ }^{4}$ \\ Erwin Bohn, ${ }^{5}$ Julia-Stefanie Frick, ${ }^{5}$ Oliver Borst, ${ }^{1}$ Patrick Münzer, ${ }^{1}$ Britta Walker, ${ }^{1}$ Justin Markel, ${ }^{2}$ Gabor Csanyi, ${ }^{6}$ Patrick J. Pagano, ${ }^{6}$ \\ Patricia Loughran, ${ }^{2,7}$ Morgan E. Jessup, ${ }^{7}$ Simon C. Watkins, ${ }^{7}$ Grant C. Bullock, ${ }^{8}$ Jason L. Sperry, ${ }^{2}$ Brian S. Zuckerbraun, ${ }^{2}$ \\ Timothy R. Billiar, ${ }^{2}$ Michael T. Lotze, ${ }^{2}$ Meinrad Gawaz, ${ }^{1}$ and Matthew D. Neal ${ }^{2}$ \\ 'Department of Cardiology and Cardiovascular Diseases, Eberhard Karls University Tübingen, Tübingen, Germany. ${ }^{2}$ Department of Surgery, University of Pittsburgh, Pittsburgh, Pennsylvania, USA. \\ ${ }^{3}$ Interfaculty Institute of Biochemistry, ${ }^{4}$ Institute of Applied Physics, and ${ }^{5}$ Institute of Medical Microbiology and Hygiene, Eberhard Karls University Tübingen, Tübingen, Cermany. ${ }^{6}$ Department of Pharmacology \\ and Chemical Biology, ${ }^{7}$ Center for Biologic Imaging, and ${ }^{8}$ Department of Pathology and University of Pittsburgh Vascular Medicine Institute, University of Pittsburgh, Pittsburgh, Pennsylvania, USA.
}

\begin{abstract}
Thrombosis and inflammation are intricately linked in several major clinical disorders, including disseminated intravascular coagulation and acute ischemic events. The damage-associated molecular pattern molecule high-mobility group box 1 (HMGB1) is upregulated by activated platelets in multiple inflammatory diseases; however, the contribution of plateletderived HMCB1 in thrombosis remains unexplored. Here, we generated transgenic mice with platelet-specific ablation of HMCB1 and determined that platelet-derived HMCB1 is a critical mediator of thrombosis. Mice lacking HMCB1 in platelets exhibited increased bleeding times as well as reduced thrombus formation, platelet aggregation, inflammation, and organ damage during experimental trauma/hemorrhagic shock. Platelets were the major source of HMCB1 within thrombi. In trauma patients, HMCB1 expression on the surface of circulating platelets was markedly upregulated. Moreover, evaluation of isolated platelets revealed that HMCB1 is critical for regulating platelet activation, granule secretion, adhesion, and spreading. These effects were mediated via TLR4- and MyD88-dependent recruitment of platelet guanylyl cyclase (GC) toward the plasma membrane, followed by MyD88/GC complex formation and activation of the cGMP-dependent protein kinase I (cCKI). Thus, we establish platelet-derived HMGB1 as an important mediator of thrombosis and identify a HMCB1-driven link between MyD88 and CC/CCKI in platelets. Additionally, these findings suggest a potential therapeutic target for patients sustaining trauma and other inflammatory disorders associated with abnormal coagulation.
\end{abstract}

\section{Introduction}

Untoward thrombus formation is associated with multiple major clinical disorders and is a leading cause of death and disability worldwide (1). Excessive platelet activation and aggregation at sites of disrupted vascular integrity typically induce thrombosis, which may result in acute vessel occlusion and ischemic events $(1,2)$. Thrombus formation is inseparably linked with inflammation, and recent emphasis on the role of platelets as sentinel innate immune cells demonstrates that platelets provide a unique link between coagulation and immune responses (3-6).

High-mobility group box 1 (HMGB1), a highly conserved, nonhistone, architectural DNA-binding nuclear protein, acts as a damage-associated molecular pattern (DAMP) molecule when released by dying cells or actively secreted by stressed cells, initiating inflammation (7-10). Although lacking a nucleus, platelets express HMGB1, and, following platelet activation, HMGB1 is both exported to the cell surface as well as released into the extracellular space (11-13). Serum/plasma levels of HMGB1 are upregulated in multiple inflammatory disease states associated with

Authorship note: Meinrad Gawaz and Matthew D. Neal are co-senior authors. Conflict of interest: The authors have declared that no conflict of interest exists. Submitted: March 2, 2015; Accepted: October 1, 2015.

Reference information: / Clin Invest. 2015;125(12):4638-4654. doi:10.1172/JCI81660. abnormal coagulation, including myocardial infarction $(14,15)$, stroke (14), sepsis (16), disseminated intravascular coagulation (DIC) (17), trauma $(18,19)$, and hemorrhagic shock (20). Moreover, the diverse biological functions of extracellular HMGB1 bear striking similarities to those assigned to activated platelets, including microvascular endothelial inflammation (8), stimulation of neutrophil extracellular trap (NET) formation $(21,22)$, leukocyte recruitment (19), and microvascular thrombosis (23), indicating that platelet-derived HMGB1 links inflammation and thrombosis.

HMGB1 signals through agonist receptors such as the receptor for advanced glycation end products (RAGE) as well as other pattern recognition receptors, including TLR2, TLR4, and TLR9 (24, 25). Platelets express the agonist receptors $(26,27)$, suggesting a potential role for HMGB1 signaling through these molecules. Particular importance is ascribed to expression of TLR4 on platelets, which mediates LPS-induced platelet aggregation and thrombus formation $(28,29)$. We have recently demonstrated that platelet TLR4 is an essential mediator of platelet activation and aggregation in the setting of hemorrhagic shock (30). Platelet TLR4 can signal via the cGMP-dependent protein kinase I (cGKI) pathway in platelets (29), which may initiate platelet activation and aggregation $(31,32)$. However, the potential role of platelet-derived HMGB1 in cGKI-driven effects in platelets, which possibly controls the link between thrombosis and inflammation, remains unknown. 
Using a transgenic mouse model that we believe to be novel with ablation of HMGB1 in platelets, we provide evidence that platelets are the major source of HMGB1 within thrombi and identify platelet-derived HMGB1 as a critical mediator for injury-induced thrombosis in vivo. HMGB1 exerts its effects via platelet TLR4, myeloid differentiation factor 88-dependent (MyD88-dependent) recruitment of guanylyl cyclase (GC) toward the platelet plasma membrane, and formation of a hitherto unrecognized complex between MyD88 and GC followed by activation of cGKI in platelets. We further uncover a critical role of platelet-derived HMGB1 in trauma and hemorrhagic shock, in a setting in which inflammation and microvascular thrombosis are intricately linked $(33,34)$.

\section{Results}

Platelet-derived HMGB1 mediates platelet aggregation and thrombosis. We used a Cre/loxP system to create transgenic mice with

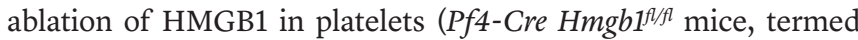
Hmgb1 Pf4 mice) and investigated the role of platelet-derived HMGB1 in hemostasis. The desired phenotype was obtained by crossing floxed Hmgb1 mice ( $H m g b 1^{f / / f l}$ mice, termed Hmgb1 Flox mice) (35) with platelet factor 4-Cre (Pf4-Cre) transgenic mice (36). Platelet-specific Hmgb1 knockout was confirmed by immunofluorescence staining (Supplemental Figure 1A; supplemental material available online with this article; doi:10.1172/JCI81660DS1) and Western blot analysis of isolated platelets (Supplemental Figure 1B). In tail vein bleeding time experiments, bleeding of Hmgb1 Pf4 mice was significantly prolonged as compared with that of $H m g b 1$ Flox control mice $(200.6 \pm 39.8$ s vs. $106.1 \pm 34.5 \mathrm{~s}, P<0.001)$ (Figure $1 \mathrm{~A})$. However, prothrombin time (PT) (Figure $1 \mathrm{~B})$, activated partial thromboplastin time (aPTT) (Figure 1C), and thrombin time (TT) (Figure 1D) were not significantly altered in Hmgb1 Pf4 mice as compared with those in control animals. Moreover, blood platelet counts of Hmgb1 Pf4 mice were comparable with those of Hmgb1 Flox control mice (data not shown).

To further evaluate the effect of platelet-specific HMGB1 deficiency on platelet function, we studied platelet aggregation in whole blood obtained from these mice. Aggregation of platelets was significantly decreased in $H m g b 1 \mathrm{Pf} 4$ mice as compared with that in $H m g b 1$ Flox mice (AUC, $38.4 \pm 5.5$ vs. $52.6 \pm 5.2$, $P<0.01$ ) (Figure 1, E and F). We next investigated the role of platelet-derived HMGB1 in thrombosis using a flow chamber system. Perfusion of blood derived from Hmgb1 Flox control mice over a collagen-coated surface with high shear rates $\left(1,700 \mathrm{~s}^{-1}\right)$ was followed by moderate formation of stable thrombi, which was significantly decreased when blood derived from Hmgb1 Pf4 mice was used (thrombus area, $49.79 \% \pm 7.3 \%$ vs. $29.86 \% \pm 6.4 \%$, $P<0.05$ ) (Figure 1G). Preincubation of blood with recombinant HMGB1 (rHMGB1) for 30 minutes was sufficient to significantly reverse these effects and induce thrombus formation in Hmgb1 Pf4 mice $(P<0.001)$, although to a lesser degree than in Hmgb1 Flox mice $(P<0.001)$. Because these observations were very suggestive, we studied the role of platelet-derived HMGB1 in an in vivo model of thrombosis. In ferric chloride-induced ( $\mathrm{FeCl}_{3}$-induced) thrombosis, blood flow in the mesenteric artery, imaged by laser Doppler velocimetry to assess thrombus formation, was significantly increased in Hmgb1 Pf4 mice 8 to 18 minutes following induction of injury as compared with that in $\mathrm{Hmgb1}$ Flox control mice $(P<0.05)$ (Figure $1 \mathrm{H})$. Moreover, the time from vessel injury to formation of stable occlusive thrombus (time to occlusion) was significantly elevated in mice with platelet-specific Hmgb1 knockout (Hmgb1 Pf4 mice) as compared with that in control mice (13.25 \pm 3.3 min vs. $18.7 \pm 1.0 \mathrm{~min}, P<0.05$ ) (Figure 1 , I and J). Isolated $\mathrm{FeCl}_{3}$-induced clots were further subjected to immunofluorescence staining (Figure 1, K and L) and Western blot analysis (Figure $1 \mathrm{M})$. CD41-positive platelet aggregates in thrombi derived from Flox control mice were substantially decreased in Hmgb1 Pf4 mice (Figure 1K). HMGB1, which was adjacent to platelet aggregates in Flox control mice, was not detected in the platelet-specific knockout animals. Next, we investigated the role of platelet-derived HMGB1 in recruitment of Ly6G-positive immune cells and formation of citrullinated histone $\mathrm{H} 3$-positive (citH3-positive) NETs (21) in thrombi. Thrombi harvested from control animals demonstrated a substantial accumulation of NETs and Ly6G-positive cell infiltrates, which was markedly reduced in Hmgb1 Pf4 mice (Figure 1L). Western blot analysis revealed a substantial loss of HMGB1 abundance in the clots taken from Hmgb1 Pf4 mice, indicating that platelets are the major source of HMGB1 within thrombi (Figure 1M).

We and others have previously shown that platelets store and, upon activation, express HMGB1 on their surfaces (12) and release it in significant amounts into the extracellular space (13). To test HMGB1 expression during thrombus formation, we perfused blood, which was previously stained with a labeled antiHMGB1 antibody, through a flow chamber and performed live imaging of the forming thrombi in vitro. Marked HMGB1 expression was detected during a 100-second investigation period (Supplemental Figure 2).

Platelet-derived HMGB1 promotes platelet aggregation and small vessel thrombosis in the lung and liver and increases inflammation during trauma and hemorrhagic shock. Having detected platelet-derived HMGB1 as a critical factor for platelet aggregation and platelet-dependent thrombus formation, we investigated platelet HMGB1 and its effects during trauma and hemorrhagic shock. In trauma patients, expression of HMGB1 on the surface of circulating platelets $\left(\mathrm{CD} 42 \mathrm{~b}^{+} \mathrm{HMGB1}^{+}\right)$was markedly, but not significantly, elevated on the same day of the event (day 1) $(P=0.13)$ and significantly elevated 1 day (day 2$)(P<0.05)$ and 2 days (day 3) $(P<0.01)$ following the event, as compared with that in healthy subjects and evaluated by flow cytometry (Figure 2A), likely due to translocation of HMGB1 to the platelet surface. Significant $(P<0.004)$ differences within the 4 subgroups were detected. In an experimental trauma/hemorrhagic shock model, consisting of soft tissue injury, bone pseudofracture, hemorrhage, and liver crush, aggregation of circulating platelets was significantly $(P<0.001)$ increased in whole blood obtained from Hmgb1 Flox control mice 30 minutes after induction of trauma (Figure 2B). This "aggregation peak" was not detected in Hmgb1 Pf4 mice, indicating a platelet HMGB1-dependent effect. Histological examination of livers and lungs revealed substantial small vessel thrombus formation and vascular congestion in Hmgb1 Flox control mice after induction of trauma/hemorrhagic shock (Figure 2C, arrows). In Hmgb1 Pf4 mice, we detected no or only very little small vessel thrombi. Moreover, immunofluorescence staining of liver and lung tissue sections, using CD41 as a platelet marker, showed substantial accu- 

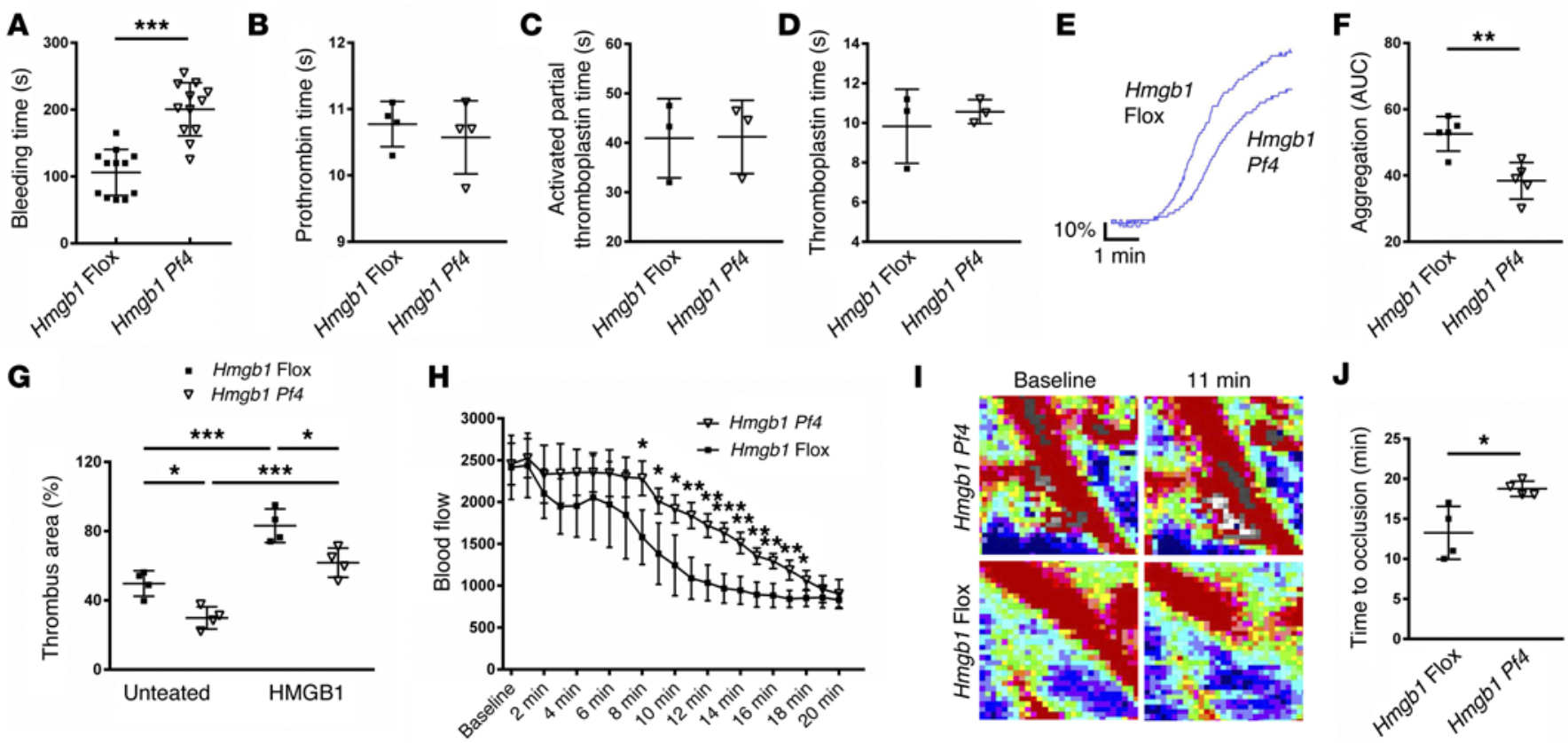

$\mathbf{J}$

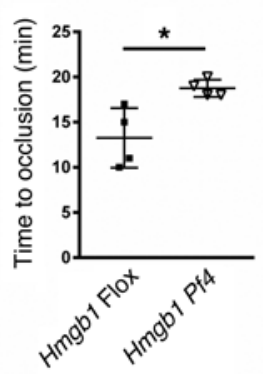

$\mathbf{K}$

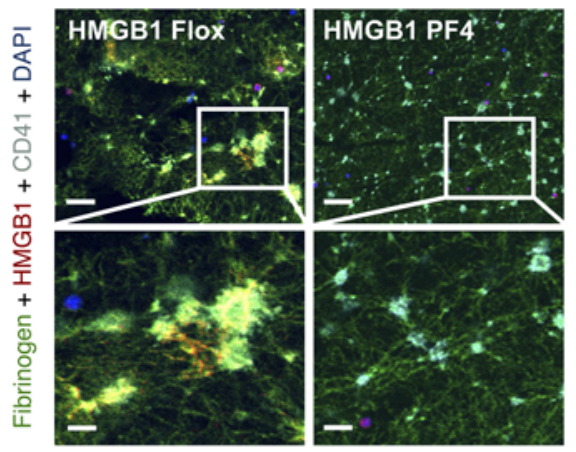

$\mathbf{L}$

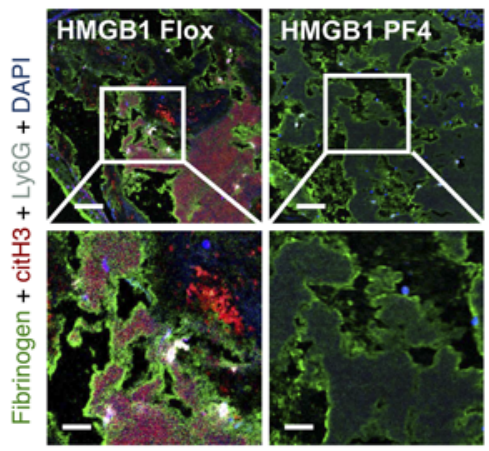

M

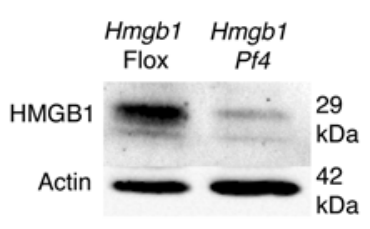

Figure 1. Platelet-derived HMGB1 promotes platelet aggregation and thrombus formation. (A) Hmgb1 Pf4 mice have prolonged bleeding time compared with Hmgb1 Flox control mice. (B) PT, (C) aPTT, and (D) TT are not altered in Hmgb1 Pf4 mice as compared with control mice. (E and F) Reduction in collagen-induced platelet aggregation in Hmgb1 Pf4 mice. (G) Blood derived from Hmgb1 Pf4 mice is less thrombogenic in a flow chamber system. Exogenous HMCB1 increases thrombus formation in blood from both $\mathrm{Hmgb1} \mathrm{Pf} 4$ and $\mathrm{Hmgb}$ 1 Flox mice. (H-J) FeCl-induced thrombus formation is inhibited in Hmgb1 Pf4 mice, with (H) improved blood flow and (I) prolonged time to vessel occlusion (as measured by laser Doppler imaging, quantified in J). (K) Immunofluorescence staining of thrombi from Hmgb1 Flox control mice demonstrates large clusters of CD41-positive platelets and surrounding deposition of HMGB1 overlying a fibrinogen network. Thrombi from Hmgb1 Pf4 animals show smaller clusters without HMGB1 expression. Scale bar: $100 \mu \mathrm{m}$ (top row), $40 \mu \mathrm{m}$ (bottom row). (L) Ly6G-positive immune cell infiltrates and citH3-positive NETs detected in FeCl-induced thrombi from Hmgb1 Flox mice but not Hmgb1 Pf4 mice. Scale bar: $100 \mu \mathrm{m}$ (top row), $40 \mu \mathrm{m}$ (bottom row). (M) Western blot of clots isolated from Hmgb1 Pf4 mice reveals almost no expression of HMGB1 as compared with control. Data show mean \pm SD from at least 3 separate experiments and (B-D) $n=3$, $(\mathbf{G}, \mathbf{H}$, and $\mathbf{J}) n=4,(\mathbf{F}) n=5$, and $(\mathbf{A}) n=12$ mice per group. (E, I, and $\mathbf{K}-\mathbf{M})$ Representative images from at least 4 mice per group. ${ }^{*} P<0.05,{ }^{* *} P<0.01$, ${ }^{* * *} P<0.001$ (Student's $t$ test in $\mathbf{A}-\mathbf{D}, \mathbf{F}, \mathbf{H}$, and J; 1-way ANOVA with Tukey's post-hoc test in $\mathbf{G}$ ).

mulation and aggregation of platelets in Hmgb1 Flox control mice after trauma/hemorrhagic shock (Figure 2D). Platelet aggregates were significantly decreased in Hmgb1 Pf4 mice.

Next, we investigated the contribution of platelet-derived HMGB1 to inflammation during trauma/hemorrhagic shock. Figure $2 \mathrm{E}$ shows representative immunofluorescence images of lung tissue sections from mice subjected to trauma/hemorrhagic shock stained with specific antibodies for citH3 and Ly6G, indicating NET formation and immune cell infiltrates. Immune cells as well as NETs, which were detected in control mice, were significantly attenuated in Hmgbl Pf4 mice. To further substantiate a critical role of platelet-derived HMGB1 in inflammation during trauma and hemorrhagic shock, we determined serum levels of circulating cytokines in Hmgb1 Flox and Hmgb1 Pf4 mice after trauma/hemorrhagic shock (Supplemental Figure 3). A significant reduction in the expression of TNF- $\alpha$, IL-6, monocyte chemoattractant protein-1 (MCP-1), and resistin was detected in Hmgb1 Pf4 mice. Measurement of serum transaminase levels (aspartate aminotransferase and alanine aminotransferase) during experimental trauma/hemorrhagic shock revealed significantly reduced hepatocellular injury in Hmgb1 Pf4 mice as compared with that in Flox control mice (Supplemental Figure 4). 
A

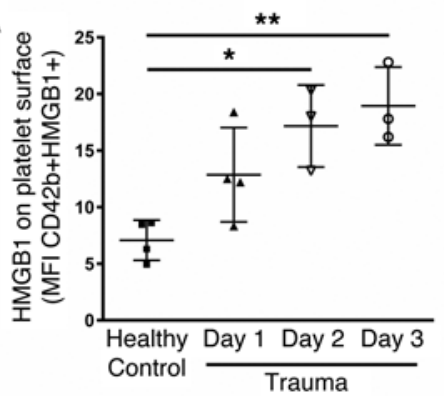

B

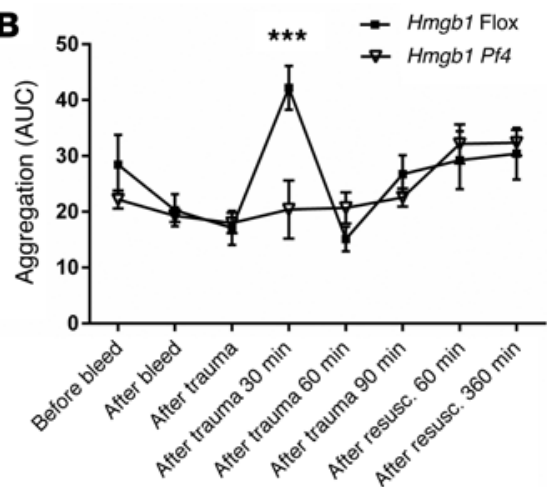

C

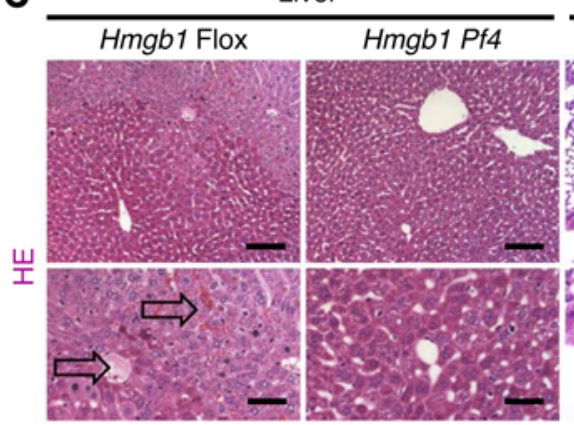

Lung

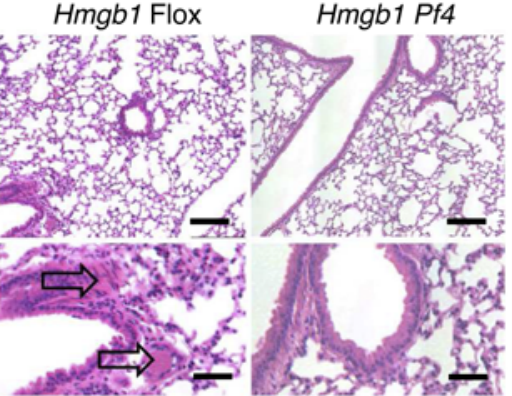

\section{D}

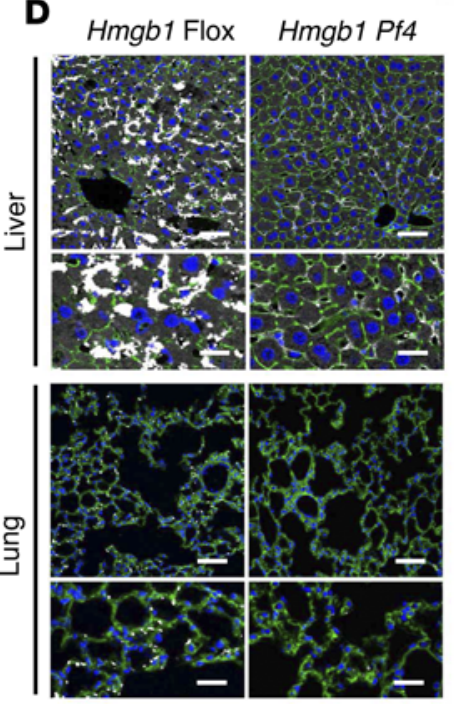

Actin + CD41 + DAPI

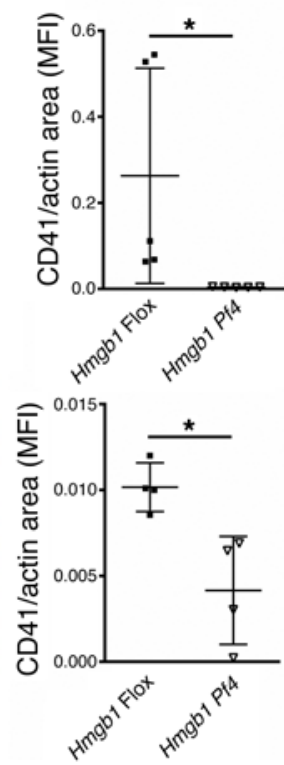

E



Figure 2. Platelet-derived HMGB1 is critical for platelet aggregation, small vessel thrombosis, NET formation, and recruitment of innate immune cells during trauma/hemorrhagic shock. (A) Expression of HMGB1 on the surface of circulating platelets is increased in trauma patients as compared with healthy subjects. (B) Systemic aggregation of circulating platelets is upregulated in Hmgb1 Flox mice 30 minutes after induction of experimental trauma/hemorrhagic shock as compared with Hmgb1 Pf4 mice. (C) Small vessel thrombi (H\&E staining) and (D) CD41-positive platelet aggregates (immunofluorescence staining and quantification of platelets) are detected in lungs and livers of Hmgb1 Flox mice but not in or only very little in Hmgb1 Pf4 mice after trauma/hemorrhagic shock. Arrows indicate small vessel thrombus formation and vascular congestion. Scale bar: $200 \mu \mathrm{m}$ (C, top row), $100 \mu \mathrm{m}$ (C, bottom row, and $\mathbf{D}$, top rows), $50 \mu \mathrm{m}$ (D, bottom rows). (E) CitH3-positive NETs and Ly6C-positive immune cell infiltrates are decreased in lungs of Hmgb1 Pf4 animals subjected to trauma/hemorrhagic shock compared with Hmgb1 Flox mice subjected to trauma/hemorrhagic shock (immunofluorescence staining and quantification). Scale bar: $10 \mu \mathrm{m}$. Data show mean \pm SD for (A) $n=4$ patients or (B-E) $n \geq 4$ mice per group from at least 3 separate experiments in all studies. ${ }^{*} P<0.05,{ }^{* *} P<0.01,{ }^{* *} P<0.001$ (Student's $t$ test in $\mathbf{B}, \mathbf{D}$, and $\mathbf{E}$; 1 -way ANOVA with Tukey's post-hoc test in $\mathbf{A})$. 
A
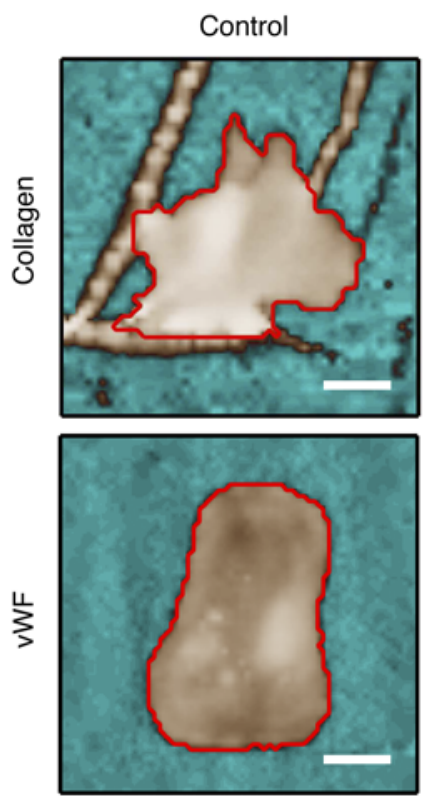
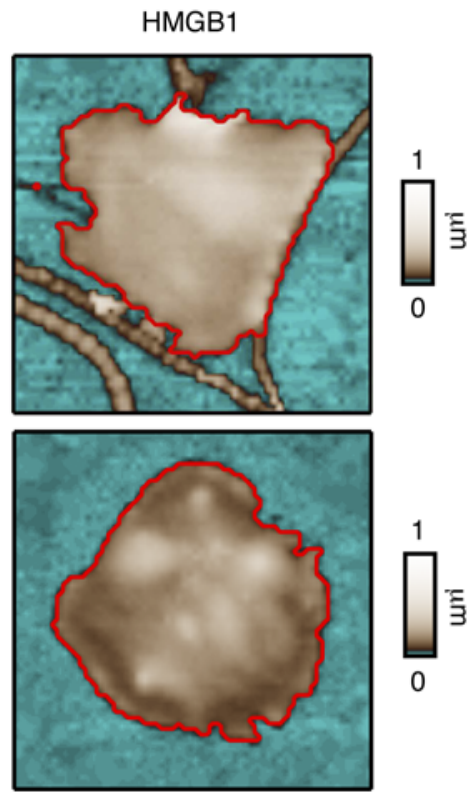
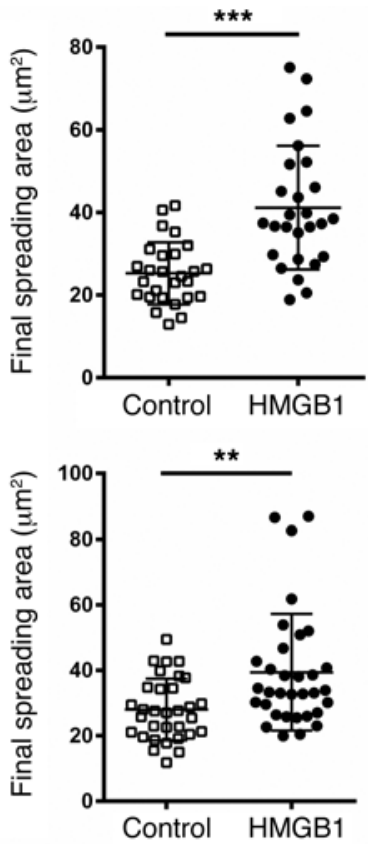

B

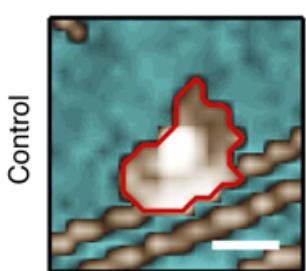

$0.14 \mathrm{~min}$

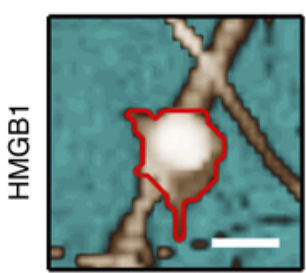

$0.16 \mathrm{~min}$

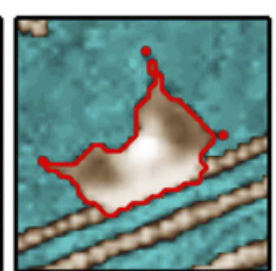

$2.1 \mathrm{~min}$

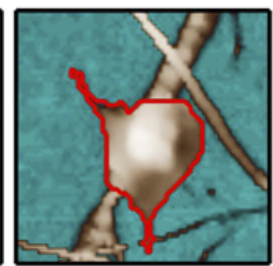

$1.5 \mathrm{~min}$

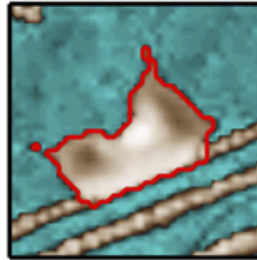

$4.8 \mathrm{~min}$

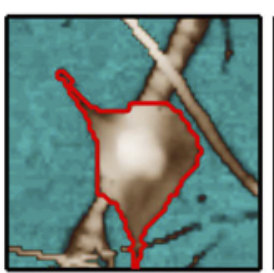

$4.9 \mathrm{~min}$

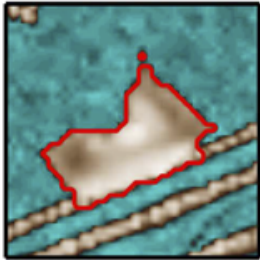

$8.8 \mathrm{~min}$

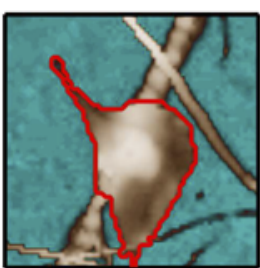

$8.3 \mathrm{~min}$

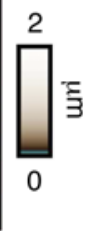

2
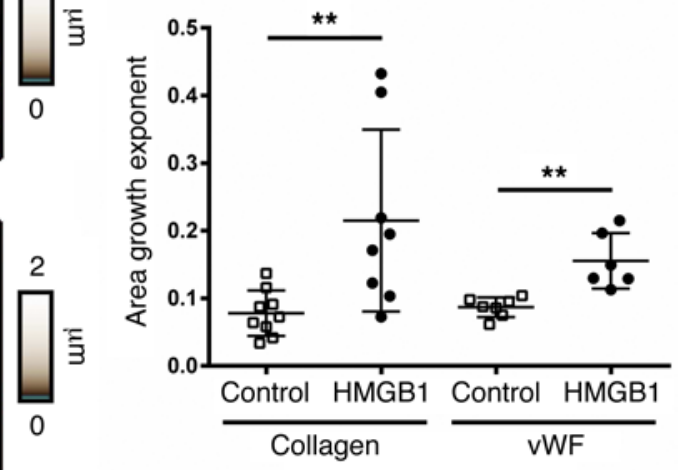

Figure 3. HMGB1 enhances platelet adhesion and spreading. (A) The addition of rHMGB1 to isolated human platelets spreading on collagen or vWF increases final platelet surface area (after 30 minutes), as investigated with SICM at single-cell level. Scale bar: $2 \mu \mathrm{m}$. (B) HMCB1 increases the speed of platelet spreading (quantified by area growth exponent) on collagen or VWF within a 9-minute investigation period. Scale bar: $3 \mu \mathrm{m}$. Data show mean \pm SD for (A) $n \geq 27$ or (B) $n \geq 6$ from at least 3 separate experiments in all studies. ${ }^{* *} P<0.01$, ${ }^{* * *} P<0.001$ (Student's $t$ test).

HMGB1 enhances platelet adhesion and spreading. Having identified platelet HMGB1 as a critical factor for injury-induced platelet aggregation, thrombosis, and inflammation, we next investigated at the single-cell level the potential contribution of HMGB1 to platelet adhesion and spreading on collagen or vWF using scanning ion conductance microscopy (SICM). Treatment with rHMGB1 significantly increased the final surface area of spread platelets on both collagen and vWF at defined endpoints (Figure 3A). Moreover, rHMGB1 treatments significantly increased the speed of platelet spreading on both collagen and vWF, as quantified by area growth exponents (Figure 3B).

HMGB1-dependent thrombosis and platelet aggregation is mediated via TLR4/MyD88. To further study the in vivo effect of HMGB1 on thrombus formation, we used the $\mathrm{FeCl}_{3}$ model, with tail vein injec- tion of either rHMGB1 ( $1 \mu \mathrm{g} / \mathrm{g}$ body weight) or PBS control prior to induction of thrombosis (Figure 4A). Injection of rHMGB1 resulted in significantly decreased time to occlusion as compared with injection of PBS control, confirming an HMGB1-induced prothrombotic effect. To test the in vivo effect of HMGB1 on platelet aggregation, we performed immunofluorescence staining of platelets (CD41) in liver and lung tissue sections after injection of rHMGB1 or PBS control into mice (Figure 4B). Significantly increased platelet sequestration in lungs and livers of the rHMGB1-injected animals was observed (Figure 4C). However, PT, aPTT, and TT were not significantly altered in rHMGB1-injected animals as compared with those in PBS controls (PT: PBS, $13.7 \pm 4 \mathrm{~s}, \mathrm{HMGB} 1,11.4 \pm 0.6 \mathrm{~s}, P=0.37$; aPTT: PBS, $15.2 \pm 5.3$ s, HMGB1, $17.3 \pm 7.2 \mathrm{~s}, P=0.71$; TT: PBS, $172 \pm$ 27.7 s, HMGB1, $168.5 \pm 36.6 \mathrm{~s}, P=0.88 ; n \geq 3$ ), which was in accor- 
A

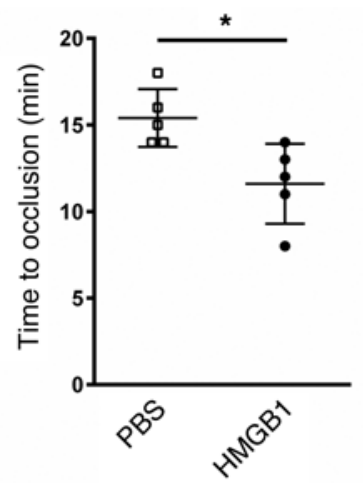

B

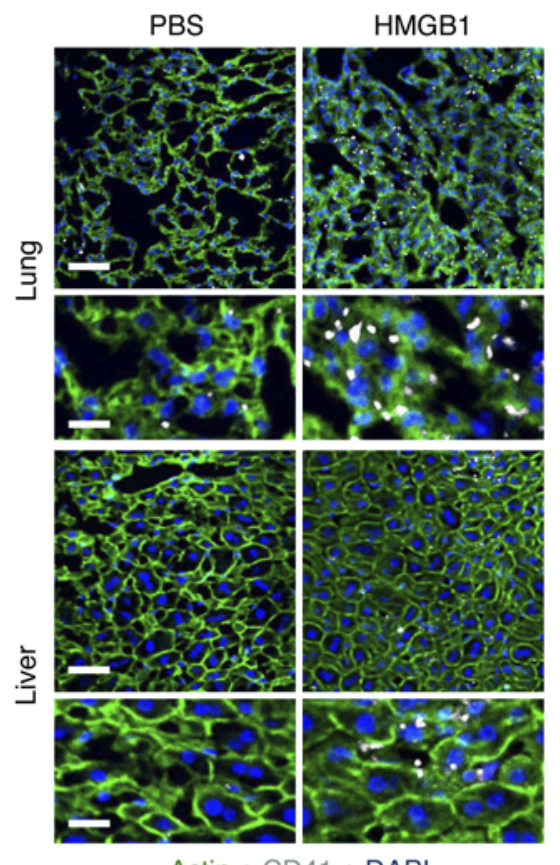

Actin + CD41 + DAPI
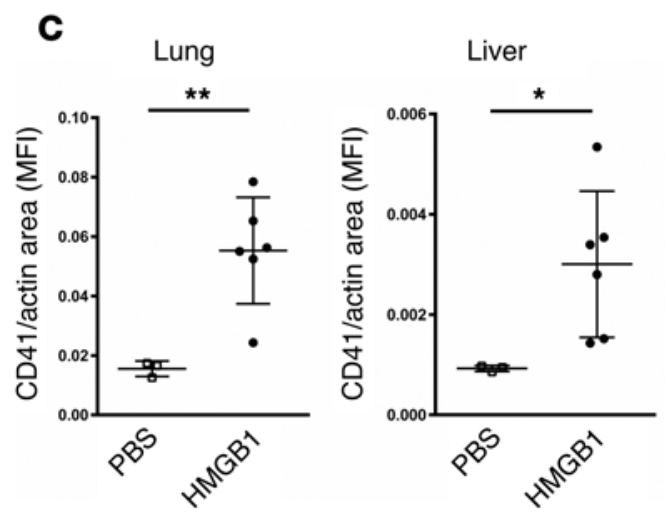

D

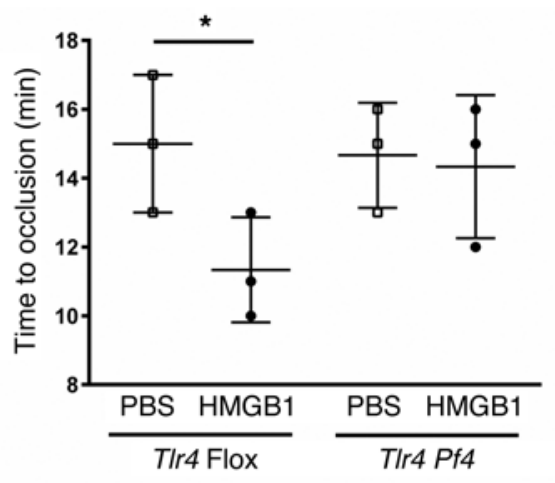

E
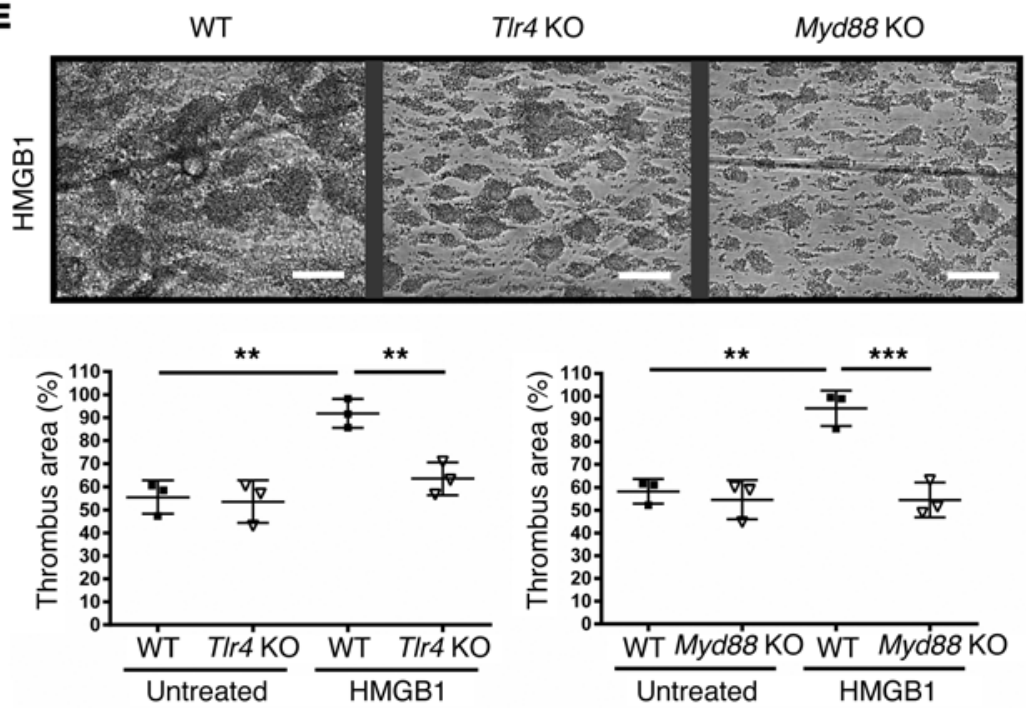

$\mathbf{F}$
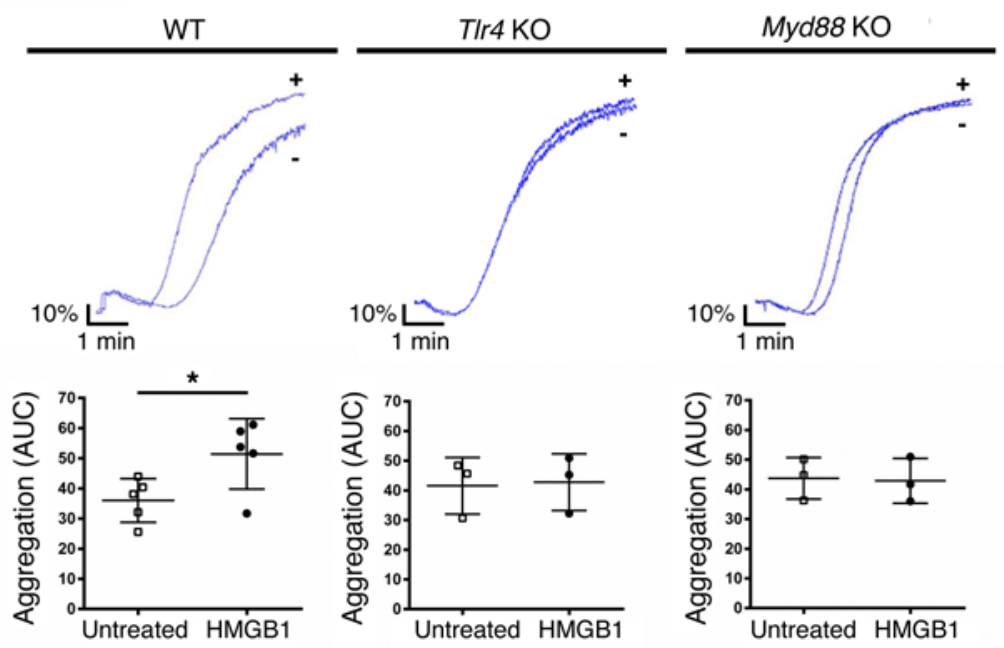

Figure 4. HMGB1 promotes thrombus formation and platelet aggregation via TLR4/MyD88. (A) Tail vein injection of rHMGB1 in C57BL/6 mice results in enhanced clot formation in a $\mathrm{FeCl}_{3}$ model (quantified as time to vessel occlusion). (B) Injection of $\mathrm{rHMCB1}$ in C57BL/6 mice increases platelet sequestration in lungs and livers. Scale bar: $100 \mu \mathrm{m}$ (top rows), $30 \mu \mathrm{m}$ (bottom rows). This is quantified in C. (D) In a FeCl ${ }_{3} \mathrm{model}_{\text {, }} \mathrm{HMCB1}$ decreases the time to vessel occlusion in TIr4 Flox control mice; this does not occur in Tlr4 Pf4 mice. (E) HMGB1 treatment of blood from WT mice induces a strong prothrombotic effect, which is reversed when repeated with blood from Tlr4 $4^{-1-}$ and Myd88 $8^{-/-}$mice. Scale bar: $70 \mu \mathrm{m}$. (F) HMCB1 treatment of blood from WT mice enhances CRP-induced platelet aggregation; this is reversed when repeated with platelets from $\mathrm{Tlr} 4^{-/-}$and $M y d 88^{-1-}$ mice. Data show mean \pm SD of the results from at least 3 separate experiments and $n \geq 3$ mice per group. ${ }^{*} P<0.05,{ }^{* *} P<0.01,{ }^{* *} P<0.001$ (Student's $t$ test in A, C, D, and F; 1-way ANOVA with Tukey's post-hoc test in E). 

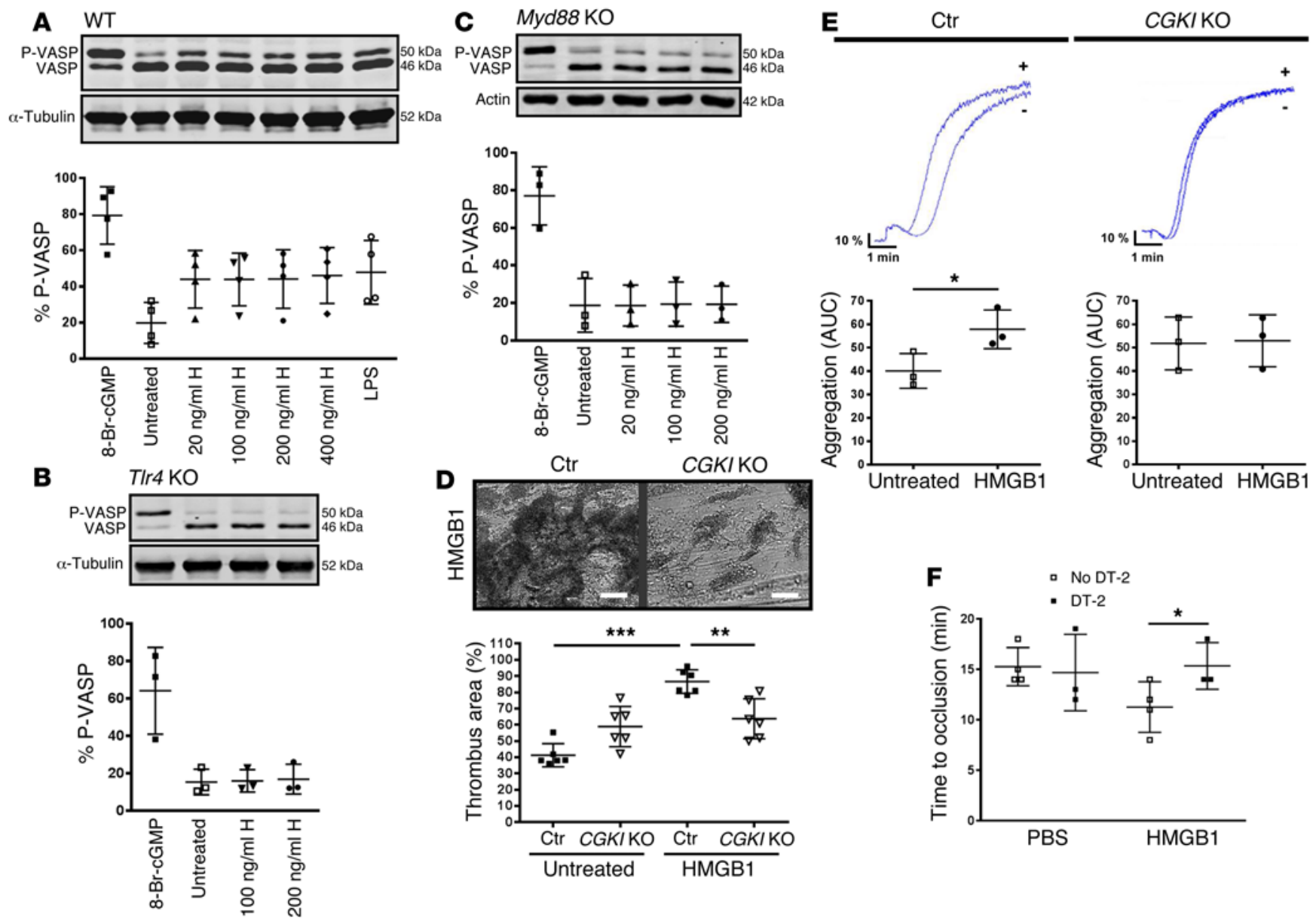

Figure 5. HMGB1 induces TLR4/MyD88-dependent CGKI activation in platelets, promoting thrombus formation and platelet aggregation. (A) Incubation of WT platelets with rHMGB1 (H) induces activation of CGKI in platelets, as validated by Western blot analysis of VASP phosphorylation

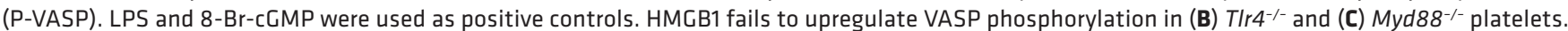
(D) The HMGB1-induced prothrombotic effect is reversed in CGKI-- blood. Scale bar: $70 \mu \mathrm{m}$. (E) The HMGB1-induced proaggregatory effect is reversed in

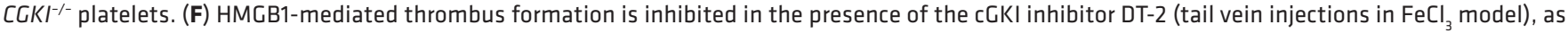
quantified by time to vessel occlusion. Data show mean \pm SD of the results from at least 3 separate experiments and $n \geq 3$ mice per group. ${ }^{*} P<0.05$, ${ }^{* *} P<0.01,{ }^{* *} P<0.001$ (Student's $t$ test in $\mathbf{E}$ and $\mathbf{F}$; 1-way ANOVA with Tukey's post-hoc test in $\mathbf{D}$ ).

dance with the coagulation tests in Hmgb1 Pf4 and Hmgb1 Flox mice described above.

Next, we analyzed the molecular mechanism underlying HMGB1-mediated thrombosis and platelet aggregation. In a $\mathrm{FeCl}_{3}$ model, we injected either rHMGB1 or PBS prior to induction of or PBS prior to induction of thrombosis in transgenic mice with ablation of TLR4 in platelets (Pf4-Cre Tlr $4^{f l / f l}$ mice, termed Tlr4 Pf4 mice) (Figure 4D). As compared with that after PBS injection, treatment of Tlr4 Flox control mice with HMGB1 resulted in a significantly decreased time to occlusion, which did not occur in Tlr4 Pf4 mice. Thus, the HMGB1-induced prothrombotic effect was mediated via platelet TLR4. Next, we performed flow chamber experiments with WT mouse blood, which was either untreated or preincubated with rHMGB1 for 30 minutes (Figure 4E). As compared with that in untreated blood, HMGB1 treatment resulted in a significant increase in thrombus formation. However, in mice lacking TLR4 (Tlr4 ${ }^{-/-}$mice) or MyD88 (Myd88 $8^{-/}$mice), the prothrombotic effect was significantly reversed ( $\mathrm{Tlr}^{-/}{ }^{-}, 30.9 \% \pm 7.7 \%$ inhibition, $P<0.01 ;$ Myd88 $/$ -, $40.7 \% \pm 8.2 \%$ inhibition, $P<0.001)$. We also studied the influence of HMGB1/TLR4/MyD88 on platelet aggregation (Figure $4 \mathrm{~F}$ ). Following activation of platelets with collagenrelated peptide (CRP), aggregation of WT platelets was significantly higher when rHMGB1 was added. However, HMGB1-mediated aggregation was almost completely abolished when repeated with $\mathrm{Tlr}^{-/-}$or $\mathrm{Myd} 88^{-/}$platelets. Furthermore, collagen-induced platelet aggregation in the presence of HMGB1 was significantly inhibited only in $\mathrm{Tlr}^{-/-}$platelets but not in $\mathrm{Tlr} 2^{-/}$or $\mathrm{Rage}^{-/}$platelets, confirming that extracellular HMGB1 promotes platelet aggregation primarily via TLR4 (Supplemental Figure 5). We also sought to define the contribution of MAPK/ERK to HMGB1-dependent platelet aggregation. In the presence of rHMGB1, the addition of U0126, a specific MEK/ERK inhibitor, significantly reduced agonist-induced platelet aggregation (Supplemental Figure 6A). Western blot analyses performed on platelet lysates from WT mice revealed that pretreatments with rHMGB1 induced phosphorylation of ERK in platelets, which was reversed in the presence of U0126 (Supplemental Figure 6B). In Tlr $4^{-/}$platelets, however, HMGB1 failed to induce ERK phosphorylation (Supplemental Figure 6C). 
A

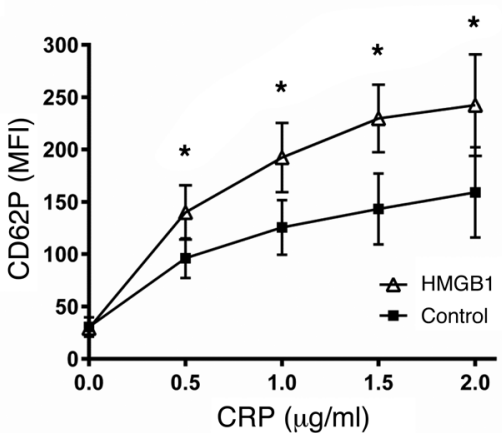

C

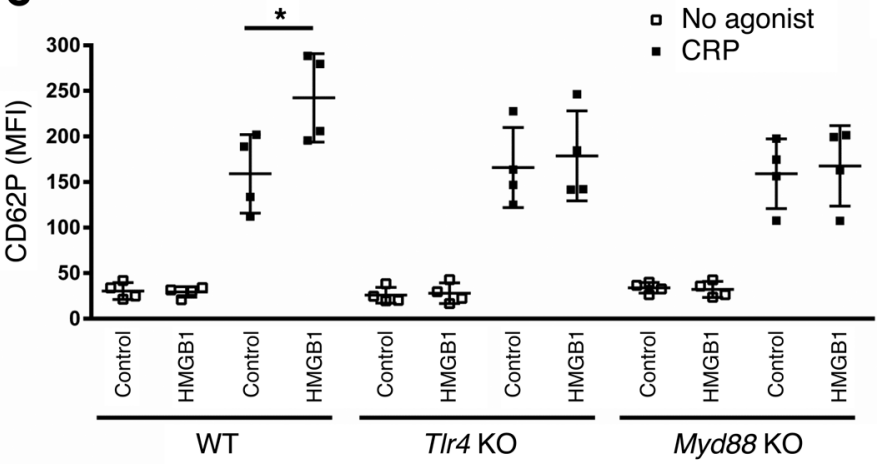

E

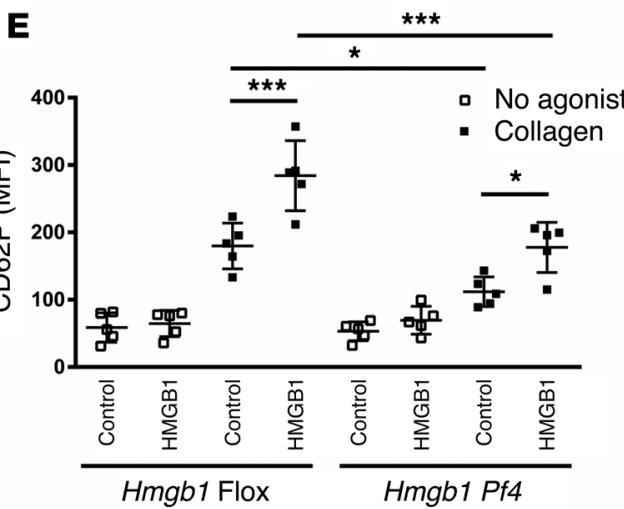

B

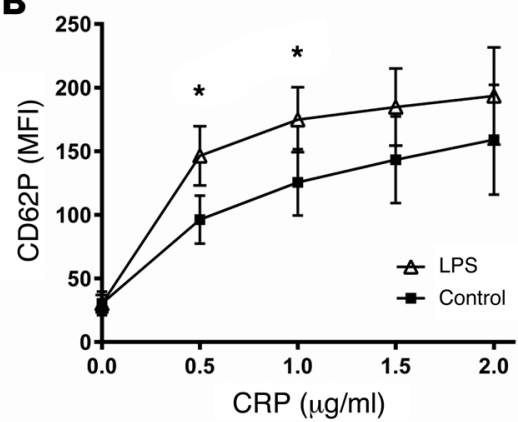

D

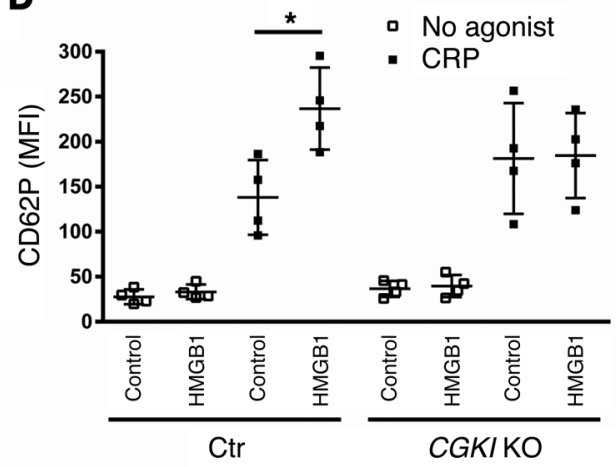

$\mathbf{F}$

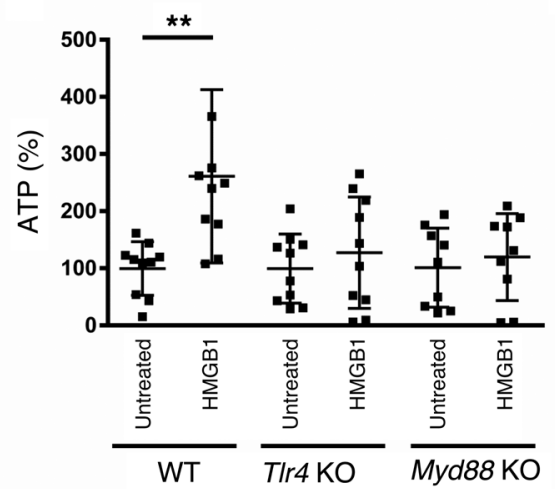

G

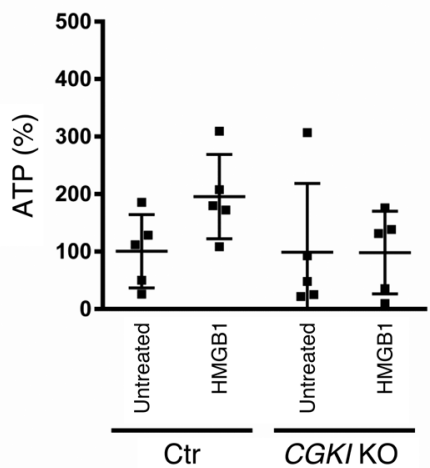

Figure 6. HMCB1 enhances agonist-induced platelet activation and granule secretion via TLR4/MyD88/cGKI. Incubation of CRP-activated platelets (0.5-2.0 $\mu \mathrm{g} / \mathrm{ml}$ ) with (A) rHMGB1 or (B) LPS enhances the expression of CD62P on the platelet surface, as evaluated by flow cytometry. (C and D) HMCB1-induced

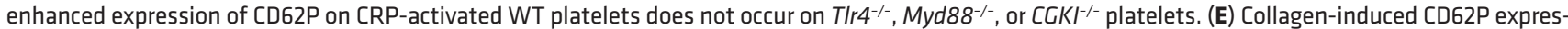
sion on the surface of HMCB1-deficient platelets is reduced, as compared with Flox control platelets. The addition of rHMCB1 enhances CD62P expression on platelets derived both from Hmgb1 Pf4 mice and Flox control mice, with stronger effects in the latter. (F and G) Incubation of CRP-activated WT platelets

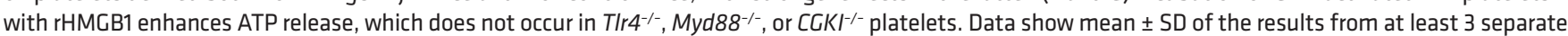
experiments and $n \geq 4$ mice per group. ${ }^{*} P<0.05,{ }^{* *} P<0.01,{ }^{* *} P<0.001$ (Student's $t$ test in $\mathbf{A}$ and $\mathbf{B} ; 1$-way ANOVA with Tukey's post-hoc test in $\mathbf{C}-\mathbf{C}$ ).

HMGB1 induces TLR4/MyD88-dependent cGKI activation in platelets, which is required for HMGB1-mediated thrombosis and platelet aggregation. The cGMP-dependent protein kinase pathway promotes LPS/TLR4/MyD88-mediated platelet aggregation (29) and activates the ERK1/2 pathway in platelets (37). To assess whether HMGB1 activates cGKI in platelets, we next investigated phosphorylation of the vasodilator-stimulated phosphoprotein (VASP), which allows monitoring of cGKI activity (38), in platelets derived from WT mice in the presence or absence of rHMGB1 or LPS by Western blot. The addition of rHMGB1 as well as LPS markedly upregulated VASP phosphorylation when compared with negative (untreated) controls
(Figure 5A). The cGMP analog 8-bromo-guanosine $3^{\prime}, 5^{\prime}$-cyclic monophosphate (8-Br-cGMP) served as a positive control due to its cGKI-activating effects and induced strong phosphorylation of VASP in platelets. However, in platelets derived from Tlr $4^{-/-}$

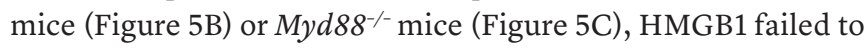
induce VASP phosphorylation, indicating that HMGB1 induces cGKI activation in platelets via TLR4/MyD88.

We have previously reported that activated platelets release significant amounts of HMGB1 into conditioned media (13). To validate that HMGB1 released by activated platelets induces activation of cGKI in platelets via TLR4, we incubated isolated WT or $\mathrm{Tlr}^{-/-}$platelets with supernatants from unactivated 

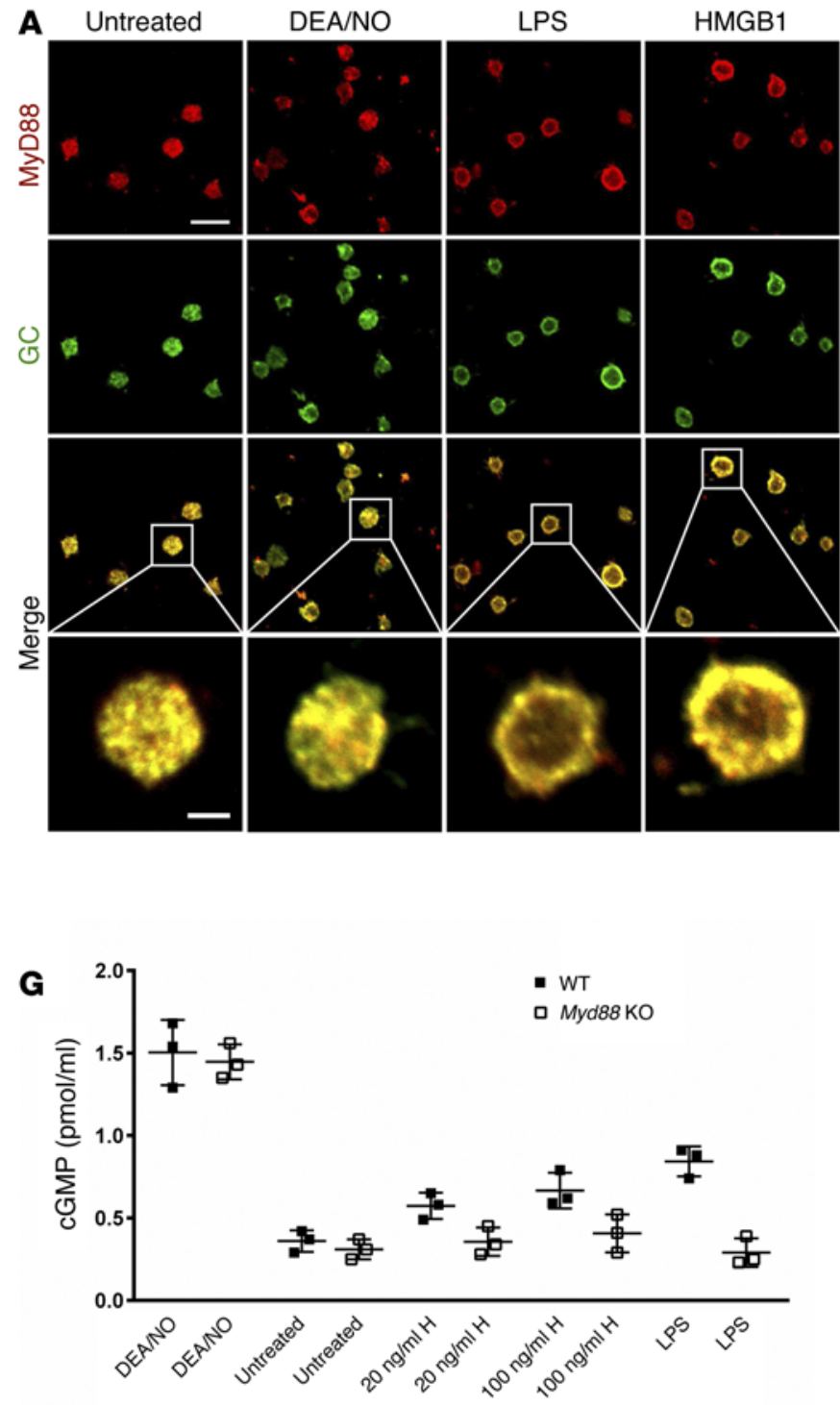

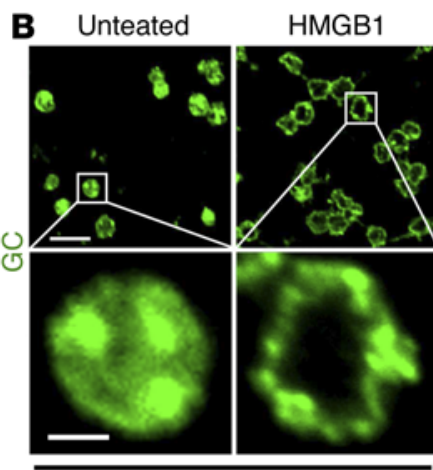

WT

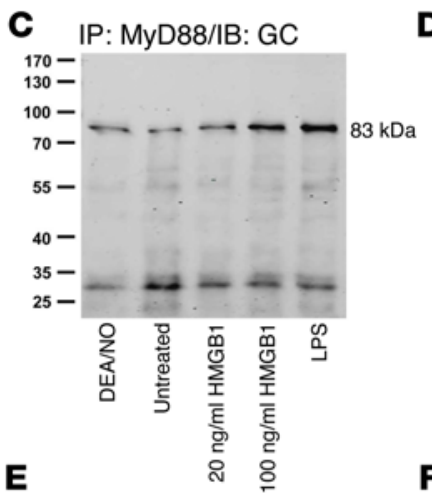

$\mathbf{E}$

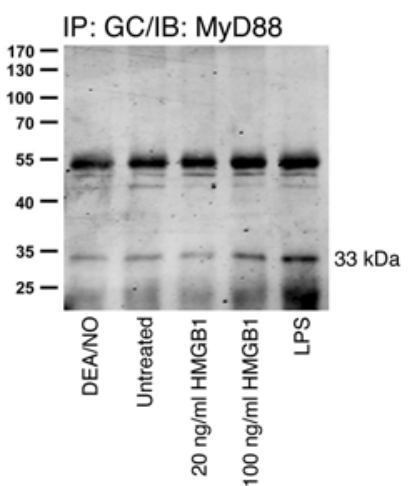

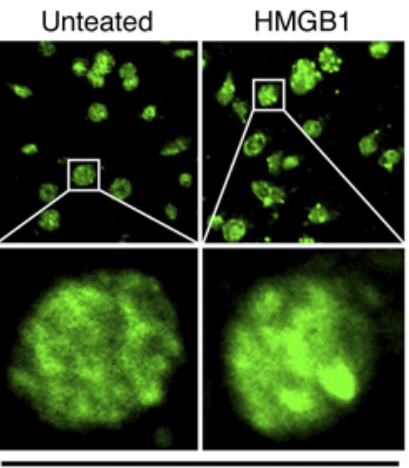

Myd88 KO

D
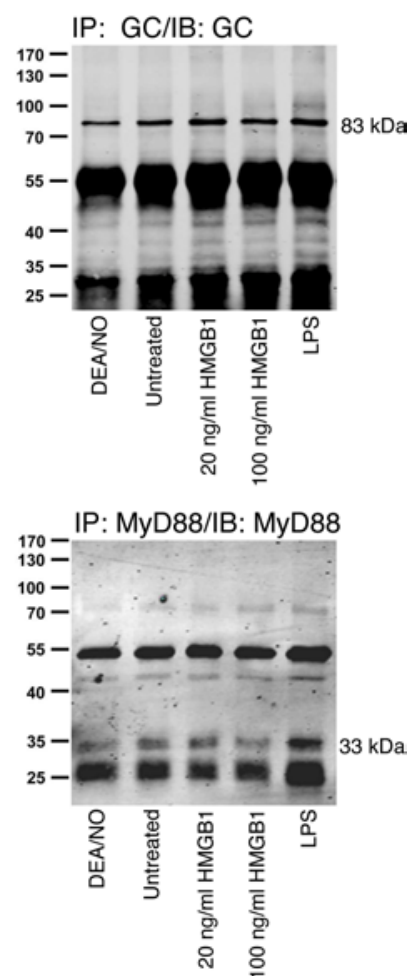

Figure 7. HMGB1 induces MyD88-dependent recruitment of GC to the platelet plasma membrane, MyD88/GC complex formation, and cGMP production in platelets. Detection of intracellular (A) MyD88 and (A and B) GC in isolated (A) human and (B) murine (WT, Myd88 ${ }^{-/-}$) platelets by immunofluorescence staining and confocal laser scanning microscopy. (A) Treatment of human platelets with HMGB1 or LPS induces translocation of MyD88 and GC toward the plasma membrane in platelets, whereas untreated and DEA/NO-treated platelets show a uniform intracellular distribution of MyD88 and GC. Scale bar: $5 \mu \mathrm{m}$ (top 3 rows), $1 \mu \mathrm{m}$ (bottom row). (B) HMGB1 also induces translocation of GC toward the plasma membrane in murine WT platelets, which does not occur in Myd88 $8^{-1-}$ platelets. Scale bar: $5 \mu \mathrm{m}$ (top row), $1 \mu \mathrm{m}$ (bottom row). (C-F) Coimmunoprecipitation studies reveal HMCB1-dependent complex formation of MyD88 and GC in murine platelets. (G) HMGB1 treatment induces CGMP production in WT platelets, which does not occur in Myd88 ${ }^{-1-}$ platelets. Data show mean $\pm S D$ of the results from at least 3 separate experiments and $n \geq 3$ mice per group.

(resting) or CRP-activated WT platelets (conditioned platelet media) (Supplemental Figure 7). Conditioned media from activated but not resting platelets induced phosphorylation of VASP in WT platelets, which was markedly reversed when activated platelets were treated with the specific HMGB1 inhibitor glycyrrhizin prior to generation of conditioned media (Supplemental Figure 7A). However, conditioned media failed to induce VASP phosphorylation in $\mathrm{Tlr}^{-/}$platelets (Supplemental Figure 7B), indicating that HMGB1 released by activated platelets induces cGKI activation in platelets via TLR4.
The potential contribution of cGKI to HMGB1-mediated platelet aggregation and thrombus formation was studied using cGKI knockout platelets $\left(\mathrm{CGKI}^{--}\right.$platelets). In flow chamber experiments, incubation of cGKI-deficient blood with rHMGB1 was followed by a significantly reduced prothrombotic effect as compared with that after treatment of control mice $(26.4 \% \pm 14.2 \%$ inhibition, $P<0.01$ ) (Figure 5D). Moreover, rHMGB1 significantly enhanced platelet aggregation in control mice, which was almost completely abolished in $C G K I^{-/}$platelets (Figure 5E). To confirm this observation in vivo, we injected mice with rHMGB1 


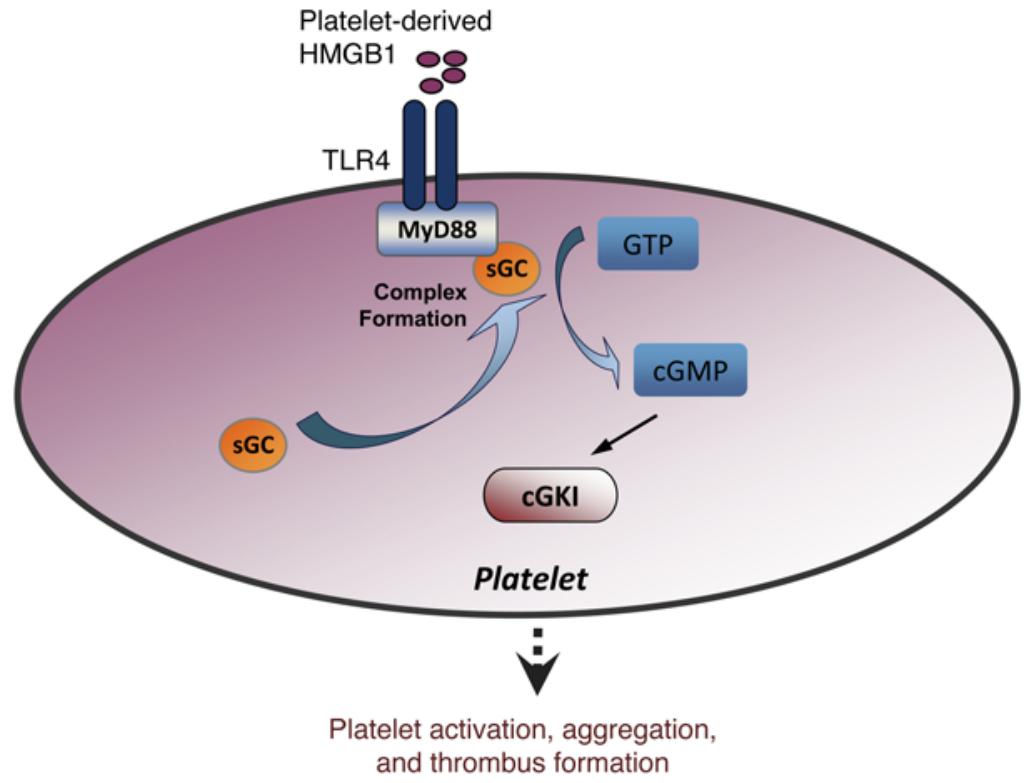

in the presence or absence of DT-2, a cGKI inhibitor (39), prior to induction of thrombosis using a $\mathrm{FeCl}_{3}$ model (Figure 4F). HMGB1 led to a reduced time to vessel occlusion, which was significantly reversed in the presence of DT- 2 .

HMGB1 enhances agonist-induced platelet activation and granule secretion via TLR4/MyD88/cGKI. We investigated the potential role of the HMGB1/TLR4/MyD88/cGKI pathway in two additional key platelet functions, i.e., platelet activation and dense granule release, which were monitored by surface expression of $\mathrm{P}$ selectin $(\mathrm{CD} 62 \mathrm{P})$ and release of ATP, respectively. CRP dosedependently induced expression of CD62P on WT platelets, which was significantly enhanced in the presence of both rHMGB1 (Figure 6A) and LPS (Figure 6B) at low concentrations of the platelet agonist. At higher CRP concentrations, however, HMGB1, but not LPS, still exerted significant effects. HMGB1-mediated upregulation of CD62P expression on activated WT platelets did not occur when $\mathrm{Tlr}^{-/-}$or $\mathrm{Myd88^{-/ }}$ platelets were used (Figure 6C). Similarly, a significant HMGB1-mediated increase of CD62P expression on activated cGKI control platelets was not observed on $\mathrm{CGKI}^{-/-}$platelets (Figure 6D). Next, we investigated the effect of platelet-specific HMGB1 ablation on platelet activation. Deletion of HMGB1 from platelets resulted in a significantly reduced collagen-induced CD62P expression (Figure 6E), suggesting a critical role for intracellular HMGB1 in addition to the effects mediated by rHMGB1. Moreover, treatment of activated HMGB1-deficient platelets with rHMGB1 significantly reversed the knockout-induced effect, although the overall effect was blunted when compared with that in Hmgb1 Flox platelets. Importantly, baseline expression of CD62P prior to agonist stimulation was affected in neither Hmgb1 Flox platelets nor Hmgb1 Pf4 platelets by rHMGB1. Similar results were obtained with ADP-activated platelets (data not shown).

Next, platelet ATP release was quantified as a marker of dense granule secretion, with similar results to CD62P expression. Treatment of CRP-activated WT platelets with rHMGB1 resulted in significantly enhanced ATP release, which did not occur when
Figure 8. Schematic overview of the molecular mechanism underlying HMGB1-driven effects in platelets. Platelet-derived HMCB1 promotes platelet activation, aggregation, and thrombus formation via TLR4- and MyD88-dependent recruitment of CC toward the platelet plasma membrane, followed by MyD88/GC complex formation, GC activation, and CGMPdependent activation of CGKI in platelets. sGC, soluble GC.

$\mathrm{Tlr}^{-/-}$or $\mathrm{Myd} 88^{-/-}$platelets were used (Figure $6 \mathrm{~F}$ ). Moreover, HMGB1 failed to increase ATP release from activated $\mathrm{CGKI}^{-/-}$platelets, whereas release from cGKI control platelets was markedly but not significantly enhanced (Figure 6G).

HMGB1 induces MyD88-dependent recruitment of GC to the platelet plasma membrane, followed by $M y D 88 / G C$ complex formation and increased cGMP production in platelets. Having identified a critical role for HMGB1/TLR4/MyD88-mediated cGKI activation in platelet activation, granule release, aggregation, and thrombus formation, we next investigated whether HMGB1-induced activation of cGKI in platelets is dependent on the second messenger cGMP. The presence of GC, a critical enzyme of the NO signaling pathway that produces CGMP, and a possible interaction of GC with MyD88 in platelets were studied. Figure 7A shows representative immunofluorescence confocal laser scanning microscopy images of permeabilized human platelets double stained with GC-specific and MyD88-specific antibodies. Both GC and MyD88 were present in the cytosol of resting as well as diethylamine NONOatetreated (DEA/NO-treated) platelets, whereas GC and MyD88 translocated toward the plasma membrane upon stimulation with rHMGB1 or LPS. HMGB1-induced translocation of GC toward the plasma membrane, which also occurred in WT platelets, was not observed in $\mathrm{Myd88^{-/ }}$ platelets, indicating a MyD88-dependent phenomenon (Figure 7B). To further analyze possible HMGB1induced MyD88/GC interactions in platelets, platelet lysates were immunoprecipitated with anti-MyD88 (Figure 7, C and F) or antiGC antibody (Figure 7, D and E) and subjected to Western blot analysis for MyD88 (Figure 7, E and F) or GC (Figure 7, C and D). Platelet preincubation with rHMGB1 dose dependently induced coprecipitation of MyD88 with GC (Figure 7C) under conditions with approximately equal expression of GC (Figure 7D). Furthermore, DEA/NO did not promote MyD88/GC complex formation, whereas LPS did (Figure 7C). The reciprocal immunoprecipitation was also performed with comparable results (Figure 7, E and F). Thus, we detected HMGB1-induced formation of a complex between MyD88 and GC in platelets.

To determine whether this MyD88/GC complex formation is followed by activation of GC, murine platelets were incubated with graded doses of rHMGB1 or LPS, and intracellular levels of cGMP were monitored by an enzyme-linked immunoassay (Figure 7G). HMGB1 and LPS markedly increased cGMP levels in WT platelets, a finding which did not occur in $M y d 88^{-/-}$platelets, proposing a MyD88-dependent phenomenon. DEA/NO served as a positive control and increased cGMP levels in a MyD88independent fashion. A schematic overview of the identified HMGB1-driven pathway in platelets is presented in Figure 8. 


\section{Discussion}

HMGB1 is an endogenous DAMP molecule (40) critical in the pathogenesis of disorders associated with abnormal coagulation, including trauma, hemorrhagic shock, and sepsis (16, 18-20, 23). A procoagulant role of HMGB1 in a thrombin-induced DIC rat model has been reported (23), and in patients with DIC, blood levels of HMGB1 correlated with platelet activation markers (41) and sepsis-related organ failure (17). Binding of rHMGB1 to activated platelets via RAGE and expression of HMGB1 in human coronary artery thrombi has recently been shown (42). Platelets store and, upon activation, express on the cell surface and release HMGB1 in significant amounts (11-13). However, the role of platelet-derived HMGB1 in thrombus formation and other platelet-dependent effects previously remained unexplored. Unexpectedly, we now establish platelets as the major source of HMGB1 within thrombi and identify platelet-specific HMGB1 as a mediator for thrombosis. Platelet activation, granule release, aggregation, intravascular thrombus formation, innate immune responses, and organ injury induced by trauma and hemorrhagic shock were critically regulated by platelet-derived HMGB1. Moreover, bleeding times were significantly prolonged in Hmgb1 Pf4 mice, despite showing no significant changes in standard coagulation tests such as PT and aPTT, indicating differential effects of platelet HMGB1 on primary hemostasis and the coagulation cascade. HMGB1 exerted the effects via TLR4- and MyD88-dependent recruitment of GC toward the platelet plasma membrane, followed by formation of a downstream complex between MyD88 and GC, activation of GC, and cGMP-dependent activation of cGKI in platelets.

A diverse collection of cytokines, chemokines, and DAMPs, such as cyclophilin A $(43,44)$, mitochondria (45), macrophage migration inhibitory factor (46), and HMGB1 (13), have been detected in platelets, and their release by platelets potentially links thrombosis with inflammation. Moreover, excessive platelet activation, e.g., during acute coronary or other thrombotic events, may result in formation of circulating monocyte-platelet aggregates, which constitutes a critical step in linking thrombosis with inflammation (47). Trauma is typically characterized by coagulation abnormalities, with significant risks of both microvascular and macrovascular thrombosis $(30,48)$, as well as early systemic elevation of HMGB1 and excessive innate immune activation $(18,49)$, which contributes to the so-called "systemic inflammatory response syndrome" and organ injury (18). The presence of HMGB1 in platelets was critical to inflammation during experimental trauma and hemorrhagic shock, as evidenced by the reduction of circulating proinflammatory cytokines as well as reduced Ly6G-positive immune cell infiltrates and NET formation in Hmgb1 Pf4 mice. Platelets are capable of inducing NETs, a finding that has been elegantly shown in a mouse model of transfusion-related acute lung injury (50). NETs promote thrombosis (51), and a critical role of HMGB1 in NET formation, mediated by RAGE (22) or TLR4/9 (21), has been reported. Further studies are necessary to obtain a better understanding of NETs induced by platelet-derived HMGB1 in injured tissues and organs.

Aggregation of circulating platelets was substantially increased 30 minutes after induction of trauma, possibly due to an early injury-induced platelet response, which did not occur in mice with HMGB1-deficient platelets. The underlying mech- anism mediating this platelet HMGB1-dependent "aggregation peak" at this specific time point is unclear, although it may represent the point of maximal HMGB1 expression and/or release by circulating platelets in mice subjected to trauma/hemorrhagic shock. Platelet-specific HMGB1 ablation was also followed by markedly reduced intravascular thrombosis and platelet sequestration within the lung and liver and hepatocellular injury during trauma/hemorrhagic shock. Further studies need to investigate whether these protective effects are due to an overall reduced inflammatory response or principally derived from an attenuated specific platelet response. In other transgenic mouse models, specific ablation of HMGB1 in hepatocytes (52) and myeloid cells (53) resulted in increased hepatocellular injury after ischemia/reperfusion and massive macrophage cell death after endotoxemia, respectively, suggesting a protective role for HMGB1 in these settings. Furthermore, we have recently demonstrated enhanced pancreatitis (35) and colonic injury (54) with targeted ablation of HMGB1 and appropriate stressors. However, detrimental effects of HMGB1, such as microvascular thrombus formation (23) as well as enhanced myocardial (55) and hepatic (56) injury after induction of ischemia/reperfusion damage, have been reported as well. Here, targeting platelet-derived HMGB1 is proposed as a new and promising strategy to limit platelet-dependent thrombosis and inflammation in organs at risk.

HMGB1 promoted platelet adhesion and spreading, as shown at the single-cell level using SICM imaging technology. The underlying mechanism by which HMGB1 exerted this effect may represent direct changes to adhesion molecule expression and/or reorganization processes of the platelet cytoskeleton (57). In addition to the increase in platelet spreading, we observed an HMGB1-mediated increase in platelet activation and granule secretion (as measured by CD62P expression and ATP release), which occurred in a TLR4/MyD88/cGKI-dependent fashion. Collectively, these modulations of platelet function triggered by HMGB1 appear to drive a prothrombotic phenotype. The HMGB1-dependent effects on platelets were mediated via platelet TLR4, indicating yet another role for platelets as sentinel innate immune regulators. Downstream of TLR4, HMGB1 initiated recruitment of GC toward the platelet plasma membrane in a MyD88-dependent fashion, followed by formation of a MyD88/ GC complex and MyD88-dependent elevation of intracellular cGMP levels in platelets, possibly as a consequence of MyD88/ GC complex formation, although this remains unproven. Further studies are required to decipher the specific mechanism that mediates HMGB1-driven MyD88/GC complex formation in platelets and its contribution to linking inflammation with hemostasis and thrombosis. The effects mediated by plateletderived HMGB1 might predominantly occur via expression of the DAMP on the surface of activated platelets, which we observed in trauma patients in this study; its release by activated platelets into extracellular space (13); or intracellular mechanisms. Here, HMGB1 released by activated platelets into conditioned media was critical for TLR4-dependent activation of cGKI in platelets, indicating at least a paracrine mechanism of signaling, although we cannot exclude an autocrine effect as well. In each case, TLR4-dependent MyD88/GC complex formation in platelets may be critical for mediating the prothrombotic effect 
of platelet-derived HMGB1, which is currently under investigation. Designing specific inhibitors that interfere with this molecular link could be of particular relevance for patients sustaining trauma and other diseases, in which inflammation and microvascular thrombosis go hand in hand $(33,34)$.

Controversy exists in the literature regarding the role of cGMP/cGKI pathway in platelet activation and aggregation, as inhibitory (58) as well as stimulatory (31) effects of cGMPdependent cGKI activation in platelets have been previously shown. A biphasic cGMP response, consisting of an early stimulatory response promoting platelet activation and aggregation, followed by a delayed inhibitory response, which is dependent on duration and concentration of cGMP levels in platelets, has been proposed as a possible explanation (31). Moreover, platelet GC plays an important stimulatory role in hemostasis and thrombosis in vivo, which has been shown in mice with a platelet-specific soluble GC knockout (59). In our study, HMGB1 upregulated cGMP levels in platelets and induced activation of platelet cGKI, as monitored by phosphorylation of VASP, which resulted in enhanced platelet aggregation and thrombus formation. Here, VASP served as a readout of cGKI activation, not necessarily as an established component of the mechanism. Moreover, HMGB1 induced phosphorylation of MAPK/ERK in platelets, which has been proposed to be downstream of cGKI signaling (37) and which occurred in a TLR4-dependent fashion in our study. Thus, our findings confirm a stimulatory effect on platelets upon activation of cGKI-dependent pathways, which may be critical for inflammation-induced abnormal platelet activation and aggregation.

Taken together, for the first time to our knowledge, we show that platelets are the major source of HMGB1 within thrombi and identify platelet-derived HMGB1 as a target for antithrombotic therapy. Interfering with HMGB1-driven complex formation of MyD88/GC and activation of cGKI in platelets may help us develop new treatment strategies for patients with abnormal coagulation.

\section{Methods}

\section{Animals}

Hmgb1 Pf4 mice were generated by crossing floxed Hmgb1 mice (Hmgb $1^{f / f l}$ mice, termed Hmgb1 Flox mice) (35) with Pf4-Cre transgenic mice (36) (The Jackson Laboratory). As both mouse strains were on a C57BL/6 background, all progeny used in our study were generated with $\mathrm{C} 57 \mathrm{BL} / 6$ mice with pure genetic backgrounds. Pf4-Cre Hmgb1 fl/fl offspring (termed Hmgb1 Pf4 offspring) were confirmed using standard genomic PCR genotyping techniques. Deletion of $H m g b 1$ was confirmed by immunofluorescence staining for HMGB1 (2 $\mu \mathrm{g} / \mathrm{ml}$ antibody) and Western Blot analysis $(1 \mu \mathrm{g} / \mathrm{ml}$ antibody) of isolated platelets using an anti-HMGB1 polyclonal antibody (rabbit IgG; ab18256, Abcam). Circulating platelet counts were assessed using an automated cell counter (Vi-CELL, Beckman Coulter). Male mice were used for experiments at an age of 8 to 12 weeks. C57BL/6 mice were purchased from The Jackson Laboratory. Global Rage knockout (Rage (-) $^{-}$, global Tlr2 knockout (Tlr2 $\left.{ }^{-/}\right)$, global Tlr4

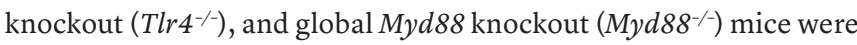
bred, genotyped, and housed at either the University of Pittsburgh (Rage ${ }^{-/-}$, Tlr2 $2^{-/-}$, and $\left.\operatorname{Tlr}^{-/-}\right)(60,61)$ or the University of Tübingen
(Tlr4 ${ }^{--}$and $\left.M y d 88^{-/-}\right)(62,63)$. Platelet-specific TLR4 knockout mice (termed Tlr4 Pf4 mice) were generated by crossing floxed Tlr4 (Tlr $4^{f / f l}$ mice, termed Tlr4 Flox mice) with Pf4-Cre transgenic mice as described previously (30). Smooth muscle-specific cGKI $\beta$ rescue mice, i.e., transgenic mice with global CGKI knockout and selective reexpression of cGKI $\beta$ in smooth muscle cells (termed CGKI KO), were generated as previously described (64). Platelets from these mice are cGKI-deficient $\left(\mathrm{CGKI}^{-/-}\right)$and were used in this study. Smooth muscle-specific cGKI $\beta$ rescue mice and litter-matched control animals that express cGKI in platelets were bred, genotyped, and housed at the University of Tübingen, Germany. We used these rescue mice to study cGKI, since conventional global cGKI knockout mice have a decreased life span, disturbed platelet activation, and other abnormal cell functions (65).

\section{Bleeding time}

Mice were anesthetized, and a 3-mm segment of the tail tip was removed with a scalpel. Tails were submerged in PBS, and bleeding was monitored as previously described (66). Bleeding time was quantified in seconds (time from initiation to cessation of blood flow).

\section{Clotting assays}

Blood was obtained via cardiac puncture from mice, and freshly generated plasma was used. Assays for PT, aPTT, and TT were performed using a hemostasis analyzer (Diagnostica Stago Start 4) according to the manufacturer's protocol. Where indicated, we injected tail veins of mice with rHMGB1 (1 $\mu \mathrm{g} / \mathrm{g}$ body weight; R\&D Systems) or PBS control 30 minutes prior to the measurements.

\section{Isolation of human and murine platelets}

Human and murine platelets were isolated as previously described (13). Venous blood from healthy volunteers was collected in acid-citratedextrose buffer and centrifuged at $430 \mathrm{~g}$ for 20 minutes. Platelet-rich plasma (PRP) was added to Tyrodes-HEPES buffer and centrifuged at $900 \mathrm{~g}$ for 10 minutes. The supernatant was discarded, and the platelet pellet was suspended in Tyrodes-HEPES buffer ( $\mathrm{pH}$ 7.4; supplemented with $\mathrm{CaCl}_{2}, 1 \mathrm{mmol} / \mathrm{l} ; \mathrm{MgCl}_{2}, 1 \mathrm{mmol} / \mathrm{l}$ ). For the preparation of murine platelets, mice were anesthetized with isoflurane and blood was drawn from the retro-orbital plexus into anticoagulated tubes. PRP was obtained by centrifugation at $260 \mathrm{~g}$ for 5 minutes, followed by another centrifugation step at $640 \mathrm{~g}$ for 5 minutes to pellet the platelets.

\section{Platelet aggregometry}

Platelet aggregation was assessed using either impedance aggregometry (whole blood) or light transmission (isolated platelets) as indicated using a ChronoLog aggregometer (Model 700). Aggregation of isolated platelets was performed at a concentration of $2 \times 10^{8}$ platelets per $\mu$ in Tyrodes-HEPES buffer ( $\mathrm{pH} 7.4$ ). After adjusting the measurement according to the manufacturer's protocol, platelets were activated with either collagen $(2 \mu \mathrm{g} / \mathrm{ml}$; ChronoLog) or CRP ( $2 \mu \mathrm{g} / \mathrm{ml}$; provided by Richard Farndale, University of Cambridge, Cambridge, United Kingdom), and platelet aggregation was measured in the presence or absence of $200 \mathrm{ng} / \mathrm{ml}$ rHMGB1 and in the presence or absence of the MEK/ERK inhibitor U0126 (10 $\mu \mathrm{M}$; Cell Signaling) for 10 minutes at $37^{\circ} \mathrm{C}$ with a stir speed of 1,200 rpm. Analysis was performed using the aggrolink- 8 software (ChronoLog). 


\section{Flow chamber}

Heparinized whole murine (C57BL/6, $\mathrm{Tlr}^{-/-}, \mathrm{Myd} 88^{-/-}, \mathrm{CGKI}^{--}$, and Hmgb1 Pf4 mice and respective control animals) or human blood from healthy volunteers was diluted 3:1 with Tyrodes-HEPES buffer ( $\mathrm{pH}$ 7.4) and perfused through a transparent flow chamber (slit depth $50 \mu \mathrm{m})$ over a collagen-coated surface $(200 \mu \mathrm{g} / \mathrm{ml})$ with high shear rates $\left(1,700 \mathrm{~s}^{-1}\right)$ for 5 minutes as described previously (46). After perfusion, the chamber was rinsed for 5 minutes by perfusion with Tyrodes-HEPES buffer, and pictures were taken from 4 to 5 different microscopic areas (using $\times 20$ optical objectives). Thrombus area was analyzed with AxioVision software (Carl Zeiss), and the mean percentage values were determined for each experimental run. In certain experiments, blood was preincubated with $400 \mathrm{ng} / \mathrm{ml}$ rHMGB1 for 30 minutes at room temperature. In another set of experiments, blood was preincubated with an Alexa Fluor 488-conjugated anti-HMGB1 monoclonal antibody $(5 \mu \mathrm{g} / \mathrm{ml}$, mouse IgG2B; clone 115603 , R\&D Systems) for 30 minutes prior to imaging the forming thrombi using a Nikon ECLIPSE Ni-E microscope (Nikon).

\section{$\mathrm{FeCl}_{3}$ thrombosis model}

$\mathrm{FeCl}_{3}$-induced thrombosis was performed on the mesenteric artery as previously described (46). Briefly, mice were anesthetized with intraperitoneal pentobarbital sodium $(50 \mathrm{mg} / \mathrm{kg}$ body weight; Oak Pharmaceuticals). After a midline abdominal incision, the mesentery was exteriorized and the mesenteric artery was injured by topical application of a Whatman filter paper soaked with $10 \% \mathrm{FeCl}_{3}$ (Sigma-Aldrich) for 30 seconds. Core temperature was maintained via heat lamp at $37^{\circ} \mathrm{C}$ and monitored by rectal probe. Vessel blood flow was monitored using laser Doppler imaging (Moor Instruments), which measures the flux of blood (blood $\times$ area $^{-1} \times$ time $^{-1}$ ). Time to occlusion was determined by visual cessation of flow. Equal areas of the control and ischemic vessel from the same anatomical regions were compared. Where indicated, mice were treated by tail vein injection with rHMGB1 $(1 \mu \mathrm{g} / \mathrm{g}$ body weight) or PBS control in the presence or absence of the cGKI inhibitor DT-2 $(0.5 \mu \mathrm{g} / \mathrm{g}$ body weight) 30 minutes prior to induction of thrombosis.

\section{Clot analysis}

Following $\mathrm{FeCl}_{3}$-induced thrombosis, the excised intravascular clot was suspended in lysis buffer (Cell Signaling), dissociated by sonication, and centrifuged at 7,000 $\times g$ for 10 minutes. The supernatant was then concentrated 10-fold with Amicon Ultra Centrifugal Filter Units (10 kDa; Merck Millipore), lysed with lysis buffer, and subjected to Western blot analysis using anti-HMGB1 polyclonal antibody $(1 \mu \mathrm{g} / \mathrm{ml}$, rabbit IgG; ab18256, Abcam) and anti-actin polyclonal antibody (1:1,000, rabbit IgG; ab1801, Abcam) as internal loading control.

For immunofluorescence staining, clots were fixed in $4 \%$ paraformaldehyde after isolation and snap frozen in liquid nitrogen. The following primary antibodies were used for staining: anti-fibrinogen polyclonal antibody ( $5 \mu \mathrm{g} / \mathrm{ml}$, rabbit IgG; ab34269, Abcam), anti-HMGB1 polyclonal antibody ( $2 \mu \mathrm{g} / \mathrm{ml}$, rabbit IgG; ab18256, Abcam), anti-CD41 monoclonal antibody ( $2 \mu \mathrm{g} / \mathrm{ml}$, rat IgG1 $;$; 553847, BD Biosciences), anti-Ly6G monoclonal antibody $(0.5 \mu \mathrm{g} / \mathrm{ml}$, rat IgG2bк; 550291, BD Biosciences), and anti-citH3 polyclonal antibody $(1 \mu \mathrm{g} / \mathrm{ml}$, rabbit IgG; ab5103, Abcam). Goat anti-rabbit IgG (1:200, Cy3 presented as red for anti-citH3 and anti-HMGB1 antibodies; 111165144, Jackson Laboratories), goat anti-rat IgG (1:200, Cy5 presented as white for
anti-CD41 and anti-Ly6G antibodies; 112175143, Jackson Laboratories), and Alexa Fluor 488-conjugated goat anti-rabbit IgG (1:100, green for anti-fibrinogen antibody; A-11008, Invitrogen) were used as secondary antibodies. Nuclear staining was carried out with DAPI (1:10,000; Abcam). Imaging was performed using either an Olympus Fluoview 1000 (Olympus) or a Nikon A1 confocal microscope (Nikon).

\section{Murine polytrauma and hemorrhagic shock model}

Hmgb1 Pf4 and Hmgb1 Flox control mice were subjected to experimental trauma and hemorrhagic shock. The animal research protocol complied with the regulation regarding the care and use of experimental animals published by the US NIH and was approved by the Institutional Animal Use and Care Committee of the University of Pittsburgh.

Bone matrix preparation for pseudofracture. Donor bones (tibia and femur) were removed from an age- and weight-matched syngenic donor, crushed using a mortar and pestle, and resuspended in PBS as described previously (67).

Polytrauma and hemorrhagic shock. Mice were anesthetized with intraperitoneal pentobarbital sodium (50 mg/ $\mathrm{kg}$ body weight) and inhaled isoflurane (Abbott Laboratories). A left groin exploration was performed, and the left femoral artery was cannulated with tapered PE-10 tubing and connected to a blood pressure transducer (Micro-Med) for continuous mean arterial pressure monitoring during the experiment (67). Induction of polytrauma was carried out in four steps. (a) Soft tissue injury was performed using an $18-\mathrm{cm}$ curved hemostat crushed with 270 psi on the bilateral lower extremities. (b) Previously prepared bone solution was injected bilaterally into the posterior muscles of each thigh at a volume of $0.15 \mathrm{cc}$ using a 20-gauge needle. (c) $30 \%$ of the total blood volume (based on 80 $\mathrm{ml}$ blood/kg body weight) was rapidly removed within 1 minute using a 1-cc syringe and a 30-gauge needle via closed cavity cardiac puncture. (d) A midline incision was made under the xyphoid, exposing the right middle lobe of the liver, which was crushed 4 times at 80 psi using a $12.5-\mathrm{cm}$ curved hemostat. Laparotomy was closed, and animals were recovered for 120 minutes. All mice were reanesthetized with i.p. sodium pentobarbital $(20 \mathrm{mg} / \mathrm{kg})$ as necessary throughout the experiment.

Resuscitation. Following the 120-minute shock period, mice were resuscitated with Ringer's lactate solution (3 times shed blood volume) over a 5-minute period. Animals were observed for indicated times after resuscitation. Mice were sacrificed under inhalational anesthesia via cardiac puncture technique. Control (sham) animals underwent anesthesia and femoral cannulation without induction of trauma or hemorrhage. Blood samples were obtained via cardiac puncture at indicated time points. Organs (liver and lung) were removed and fixed in $2 \%$ paraformaldehyde for immunohistochemistry and immunofluorescence.

\section{Measurement of serum transaminase levels and cytokine array following trauma and hemorrhagic shock}

To assess hepatic function and cellular injury following trauma/hemorrhagic shock, plasma levels of aspartate aminotransferase and alanine aminotransferase were measured using the Dri-Chem 7000 Chemistry Analyzer (Heska). To quantify changes in inflammation following trauma/hemorrhagic shock, blood was collected at the time of sacrifice and a cytokine array was performed using a colorimetric mouse cytokine ELISA plate array (Signosis) according to the manufacturer's protocol. 


\section{Histopathology}

Paraformaldehyde-fixed lung and liver samples derived from Hmgb1 Pf4 and Hmgb1 Flox mice during experimental trauma/hemorrhagic shock were processed in a Shandon Excelsior ES tissue processor (Thermo Scientific) with alcohol dehydration and xylene infiltration. Standard H\&E staining was performed to detect injured areas and intravascular thrombosis.

\section{Detection of platelets, immune cells, and NETs in liver and/or lung with immunofluorescence staining}

Immunofluorescence staining of paraffin-embedded tissue sections of lung and liver were carried out with Hmgb1 Pf4 and Hmgb1 Flox mice during experimental trauma/hemorrhagic shock and with C57BL/6 mice that received tail vein injections of either rHMGB1 $(1 \mu \mathrm{g} / \mathrm{g}$ body weight) or PBS control. Stainings were performed using anti-F-actin monoclonal antibody ( $2 \mu \mathrm{g} / \mathrm{ml}$, mouse IgM; ab205, Abcam) and antiCD41 polyclonal antibody ( $2 \mu \mathrm{g} / \mathrm{ml}$, rabbit IgG; ab63983, Abcam) or anti-Ly6G monoclonal antibody $(0.5 \mu \mathrm{g} / \mathrm{ml}$, rat IgG2bк; 550291, BD Biosciences) and anti-citH3 polyclonal antibody $(1 \mu \mathrm{g} / \mathrm{ml}$, rabbit IgG; ab5103, Abcam). Stainings were further processed as described above. Quantification was performed using a ratio of CD41 to total actin, LyG6 to total DAPI, or citH3 to total DAPI.

\section{Human sample collection inclusion and exclusion criteria for trauma patients}

Inclusion. Characteristics for inclusion include blunt traumatic injury; intact cervical spinal cord; patient arrival $\leq 6$ hours of injury; admission to intensive care unit (ICU) from the emergency department; and at least one of the following: lactate $\geq 2.5$; base deficit $\geq-3$; hypotension, with systolic blood pressure $<90 \mathrm{mmHg}$ before arriving at the hospital or trauma bay; receiving blood in trauma bay; or requirement for rapid transport to the operating room with trauma, prior to CT.

Exclusion. Characteristics for exclusion include age $<18$; anticipated survival $<24$ hours; anticipated survival $<28$ days due to preexisting condition; isolated traumatic brain injury (Glascow coma score $<8$ after ICU admission) and brain CT abnormality within 12 hours of injury; inability to obtain consent from the subject or legally authorized representative; preexisting immunosuppression; transplant recipient; chronic high doses of steroids $(>20 \mathrm{mg}$ prednisone equivalents per day); admission to the ICU primarily for substance withdrawal; known HIV-positive status and CD 4 count $<200$ cells per $\mathrm{mm}^{3}$; or inability to obtain first blood sample within 6 hours of injury.

Flow cytometric evaluation of HMCB1 and CD62P expression on platelets Blood samples from trauma patients and healthy volunteers were obtained from the antecubital vein and collected in citrate phosphate dextrose adenine, diluted 1:30 with PBS, and incubated with APCconjugated anti-CD42b monoclonal antibody (mouse IgG1к; 17-042942, eBioscience) and Alexa Fluor 488-conjugated anti-HMGB1 monoclonal antibody (mouse IgG2B; clone 115603, R\&D Systems) or respective isotype control antibodies for 30 minutes. Flow cytometry was carried out with a FACSCanto flow cytometer using DIVA software (BD Biosciences). Platelets were gated based on their characteristic scatter properties and expression of CD42b. Platelet surface expression of HMGB1 was determined by HMGB1 mean fluorescence intensity of CD42b-positive events. For murine studies, platelets were isolated from blood derived from transgenic mice and controls as described above and indicated. Resting and activated (CRP, 0.5-2 $\mu \mathrm{g} / \mathrm{ml}$; collagen, $2 \mu \mathrm{g} / \mathrm{ml}$; ADP, $30 \mu \mathrm{M}$; SigmaAldrich) platelets were treated with either LPS ( $5 \mu \mathrm{g} / \mathrm{ml}$; Sigma-Aldrich) or rHMGB1 (200 ng/ml) or control (PBS) for 30 minutes. Samples were then incubated with either FITC-conjugated anti-CD62P monoclonal antibody (rat LEW IgG1 $\lambda$; 553744, BD Biosciences) or APC-conjugated antiCD62P monoclonal antibody (mouse IgG1к; 17-0626-82, eBioscience).

\section{ATP release from platelets}

ATP release was determined to study secretion of platelet dense granules. Isolated murine platelets were adjusted to a concentration of $2.5 \times 10^{8}$ per $\mu \mathrm{l}$ and activated with CRP $(3 \mu \mathrm{g} / \mathrm{ml})$. After calibration of one sample with ATP standard (ChronoLog), ATP concentrations were determined using the ChronoLume luciferin assay (ChronoLog) on a Lumi-Aggregometer (model 700; ChronoLog) according to the manufacturer's protocol.

\section{SICM}

Isolated human platelets were added to a culture plate (Cellstar, 627160, Greiner Bio-One) coated with either collagen $(100 \mu \mathrm{g} / \mathrm{ml}$; Takeda Pharma) or vWF (10 $\mu \mathrm{g} / \mathrm{ml}$; Novus Biologicals) and allowed to adhere and spread for 30 minutes in the presence or absence of 200 ng/ml rHMGB1. High-resolution SICM topography images were obtained using a custom-built setup in back-step/hopping mode (57). The scan area was typically set to $10 \times 10 \mu \mathrm{m}^{2}$, and a pixel resolution of between $20 \times 20$ and $64 \times 64$ was chosen, depending on the required time resolution. Platelet-spreading areas were calculated by custom-written software. Briefly, the platelets were manually selected in the SICM images, and the area of all pixels with height values above $50 \mathrm{~nm}$ was calculated. The dynamic platelet-spreading behavior was determined with time-lapsed SICM imaging of adhering platelets. To obtain platelet area growth exponents, the platelet-spreading area was calculated as a function of time and fit with a power law distribution. A larger area growth exponent corresponds to a larger relative increase of spreading area in a defined time interval. The power-law behavior of cell area during spreading has been described previously for platelets using reflection interference contrast microscopy (68).

\section{Immunoblot for phosphorylation of ERK}

Isolated murine platelets derived from WT or $\mathrm{Tlr}^{-/-}$mice $\left(3 \times 10^{8}\right.$ platelets per $\mathrm{ml}$ ) were kept untreated or incubated with 100 to $200 \mathrm{ng} / \mathrm{ml}$ rHMGB1 for 15 minutes in the presence or absence of the MEK/ERK inhibitor U0126 $(10 \mu \mathrm{M})$. Processed samples were washed with PBS and lysed in immunoprecipitation buffer (Tris pH 7.4, 15 mM; NaCl, 155 $\mathrm{mM}$; EDTA, $1 \mathrm{mM}$; sodium azide, $0.08 \mathrm{mM}$ ) supplemented with protease/phosphatase inhibitor cocktail (Cell Signaling) for 60 minutes at $4^{\circ} \mathrm{C}$. Protein concentrations in cell lysates were determined with Bradford protein assay (Bio-Rad), and $40 \mu \mathrm{g}$ of protein was resolved by $8.5 \%$ SDS-polyacrylamide gel electrophoresis. Immunoblotting onto PVDF membranes (Immobilon P; Millipore) was performed using the SemiDry Transfer Cell System (Peqlab). Membranes were incubated overnight at $4^{\circ} \mathrm{C}$ with $\mathrm{p} 44 / 42 \mathrm{MAPK}$ (ERK1/2) monoclonal antibody (1:500; rabbit IgG; 9102, Cell Signaling) or phospho-p44/42 MAPK (ERK1/2) monoclonal antibody (1:1,000; rabbit IgG; 9101, Cell Signaling). For detection, corresponding secondary fluorochrome-labeled antibodies and the Odyssey infrared imaging system (LI-COR) were used.

\section{Western blot analysis of VASP phosphorylation}

Isolated murine platelets derived from WT, $\mathrm{Tlr}^{-/-}$, and $\mathrm{Myd} 8^{-/-}$mice 
$\left(3 \times 10^{8}\right.$ platelets per $\left.\mathrm{ml}\right)$ were kept untreated or incubated with graded doses of rHMGB1 (20-400 ng/ml) or LPS ( $5 \mu \mathrm{g} / \mathrm{ml}$; Sigma-Aldrich) for 15 minutes or the cGMP analog 8-Br-cGMP (100 $\mu \mathrm{M}$; Biolog) for 5 minutes at room temperature. In certain experiments, platelets were treated with conditioned media derived from resting or CRPactivated $(5 \mu \mathrm{g} / \mathrm{ml})$ WT platelets in the presence or absence of the specific HMGB1 inhibitor glycyrrhizin (10 $\mu \mathrm{g} / \mathrm{ml}$; Merck Millipore) (13). The preparations were then centrifuged at $640 \mathrm{~g}$ for 5 minutes, pellets were lysed in immunoprecipitation buffer, and protein concentrations were determined as described above. $40 \mu \mathrm{g}$ of protein was resolved by 8.5\% SDS-PAGE, and immunoblotting onto PVDF membranes was performed as described previously. Membranes were incubated overnight with anti-VASP monoclonal antibody (1:1,000, rabbit IgG; 3132, Cell Signaling), and anti-tubulin monoclonal antibody (1:1,000, mouse IgG1; 3873, Cell Signaling) or anti-actin polyclonal antibody (1:1,000, rabbit IgG; ab1801, Abcam) was used as internal loading control. Antibody binding was detected with corresponding secondary fluorescence-labeled antibodies and the Odyssey infrared imaging system. Densitometric analysis was performed with Image J software.

Immunofluorescence staining of intracellular MyD88 and GC in platelets Human or murine platelets were isolated as described above $\left(10^{7}\right.$ platelets per ml) and incubated with the NO-donor DEA/NO (1 $\mu \mathrm{M}$; Axxora) for 5 minutes, LPS ( $5 \mu \mathrm{g} / \mathrm{ml}$ ) for 15 minutes, or rHMGB1 $(200 \mathrm{ng} / \mathrm{ml})$ for 15 minutes. Untreated isolated platelets served as controls. Subsequently, platelets were fixed with $1 \%$ paraformaldehyde, applied to $0.01 \%$ polyL-lysine-coated coverslips, and permeabilized with $0.3 \%$ Triton X-100. After blocking with 1\% BSA-PBS for 1 hour, cells were incubated overnight at $4^{\circ} \mathrm{C}$ with anti-GC $\alpha 1$ polyclonal antibody $(10 \mu \mathrm{g} / \mathrm{ml}$; rabbit IgG; ab50358, Abcam). After washing with PBS plus 0.3\% Triton X-100 plus $0.1 \%$ Tween-20, platelets were incubated with Alexa Fluor 488-tagged goat anti-rabbit IgG (1:100; A-11008, Invitrogen) for 2 hours at room temperature. Following another washing step, platelets were incubated with anti-MyD88 polyclonal antibody $(2 \mu \mathrm{g} / \mathrm{ml}$; goat IgG; sc-8196, Santa Cruz), washed, and incubated with Alexa Fluor 568-tagged donkey antigoat IgG (1:100; A-11057, Invitrogen). The corresponding IgG antibodies (Santa Cruz) served as control. The coverslips were mounted with Antifade Fluorescence Mounting Medium (Dako). Confocal immunofluorescence analysis was performed using a LSM510 META confocal laser scanning microscope and ZEN 2012 imaging software (Carl Zeiss).

\section{Coimmunoprecipitation studies}

Isolated murine platelets $\left(10^{9}\right.$ platelets per $\left.\mathrm{ml}\right)$ were kept untreated or incubated with rHMGB1 (20 or $100 \mathrm{ng} / \mathrm{ml}$ ) or LPS ( $5 \mu \mathrm{g} / \mathrm{ml})$ for 15 minutes or DEA/NO $(1 \mu \mathrm{M})$ for 5 minutes at room temperature. The preparations were then centrifuged at $640 \mathrm{~g}$ for 5 minutes, and the pellets were lysed in immunoprecipitation buffer supplemented with protease/phosphatase inhibitor cocktail for 60 minutes at $4^{\circ} \mathrm{C}$. Protein concentrations in platelet lysates were determined, and 90 to $100 \mu \mathrm{g}$ of protein was used for the immunoprecipitation carried out overnight at $4^{\circ} \mathrm{C}$ with anti-MyD88 polyclonal antibody $(2 \mu \mathrm{g} / \mathrm{ml}$, goat IgG; sc- 8196 , Santa Cruz) or anti-GC $\alpha 1$ polyclonal antibody $(8 \mu \mathrm{g} / \mathrm{ml}$, rabbit IgG; ab50358, Abcam) and the respective IgG controls followed by 2 hours of incubation with washed sepharose beads (GE Healthcare) at $4^{\circ} \mathrm{C}$. Samples were washed 4 times in immunoprecipitation buffer, solubilized in Laemmli buffer, and run on a 8.5\% SDS-PAGE gel for detection of GC (83 kDa) or MyD88 (33 kDa).

\section{cGMP levels in platelets}

Isolated platelets from $\mathrm{Myd} 88^{-/-}$and WT mice were kept untreated or incubated with rHMGB1 (20 or $100 \mathrm{ng} / \mathrm{ml})$, LPS $(5 \mu \mathrm{g} / \mathrm{ml})$, or DEA/ NO $(1 \mu \mathrm{M})$ for 5 minutes. cGMP concentrations were determined using a cGMP Enzyme-Linked Immunoassay Kit (cGMP Direct Biotrak EIA; GE Healthcare) according to the manufacturer's instructions.

\section{Statistics}

All data are presented as mean \pm SD for $n \geq 3$ unless stated otherwise. Statistical significance was determined with the 2-tailed Student's $t$ test or 1-way ANOVA with Tukey's post-hoc test using Graph Pad Prism software (GraphPad). A P value of less than 0.05 was considered significant.

\section{Study approval}

Animal handling and care complied with published regulations by the US NIH and German law for the protection of animals, and experiments were approved by the local authorities (the Institutional Animal Use and Care Committee of the University of Pittsburgh and Regierungspräsidium Tübingen). Healthy volunteer and trauma patient blood samples were used following approval of the Institutional Review Board of the University of Pittsburgh (PRO 08010232). Written informed consent was received from participants prior to inclusion in the study.

\section{Author contributions}

SV, RF, EB, GCB, JLS, BSZ, TRB, MTL, MG, and MDN designed the research study. SV, RB, QC, SF, JR, PM, BW, JM, GC, PL, MEJ, SCW, and JLS conducted experiments. SV, RB, QC, SF, RF, JR, TES, J-SF, PM, BW, JM, GC, PL, MEJ, SCW, and JLS acquired the data. SV, RB, QC, JR, TES, OB, GC, PJP, PL, MEJ, GCB, BSZ, MTL, and MDN analyzed the data. SV, RF, TES, EB, J-SF, OB, PJP, SCW, JLS, BSZ, TRB, MTL, MG, and MDN provided reagents. SV, RB, QC, JR, TES, PJP, PL, GCB, BSZ, TRB, MTL, MG, and MDN wrote the manuscript.

\section{Acknowledgments}

This work was supported by the Deutsche Forschungsgemeinschaft (DFG) Klinische Forschergruppe 274 (VO 2126/1-1 to S. Vogel, GA 381/10-2 to M. Gawaz, FE 438/7-1 to R. Feil, and SCHA 1264/22 to T.E. Schäffer), the NIH (P50GM053789 to T.R. Billiar; 1S10OD019973-01, to S.C. Watkins for the Olympus Fluoview 1000 and the Nikon A1 Confocal Microscopes), the Trans-Agency Consortium for Trauma-Induced Coagulopathy (UM1 HL12087701 to B.S. Zuckerbraun), an institutional grant (to M.D. Neal) from UL1 TR000005 (to the University of Pittsburgh Clinical and Translational Science Institute), University of Pittsburgh Cancer Institute core support from the National Cancer Institute (P30CA047904), the American Association for the Surgery of Trauma Research Scholarship (to M.D. Neal), the Department of Surgery of the University of Pittsburgh, and the Department of Cardiology and Cardiovascular Diseases of the University of Tübingen.

Address correspondence to: Brian S. Zuckerbraun, Department of Surgery, University of Pittsburgh, F1200 PUH 200 Lothrop St., Pittsburgh, Pennsylvania 15213, USA. Phone: 412.864.553; Email: zuckerbraunbs@upmc.edu.

Sebastian Vogel's present address is: Department of Surgery, University of Pittsburgh, Pittsburgh, Pennsylvania, USA. 
1. Jackson SP. Arterial thrombosis - insidious, unpredictable and deadly. Nat Med. 2011;17(11):1423-1436.

2. Ruggeri ZM. Platelets in atherothrombosis. Nat Med. 2002;8(11):1227-1234.

3. Gawaz M, Langer H, May AE. Platelets in inflammation and atherogenesis. JClin Invest. 2005;115(12):3378-3384.

4. Gawaz M, Vogel S. Platelets in tissue repair: control of apoptosis and interactions with regenerative cells. Blood. 2013;122(15):2550-2554.

5. Morrell CN, Aggrey AA, Chapman LM, Modjeski KL. Emerging roles for platelets as immune and inflammatory cells. Blood. 2014;123(18):2759-2767.

6. Nurden AT. Platelets, inflammation and tissue regeneration. Thromb Haemost. 2011;105(suppl 1):S13-S33.

7. Andersson U, Rauvala H. Introduction: HMGB1 in inflammation and innate immunity. J Intern Med. 2011;270(4):296-300.

8. Fiuza $C$, et al. Inflammation-promoting activity of HMGB1 on human microvascular endothelial cells. Blood. 2003;101(7):2652-2660.

9. Scaffidi P, Misteli T, Bianchi ME. Release of chromatin protein $\mathrm{HMGB} 1$ by necrotic cells triggers inflammation. Nature. 2002;418(6894):191-195.

10. Vogel S, et al. Necrotic cell-derived high mobility group box 1 attracts antigen-presenting cells but inhibits hepatocyte growth factormediated tropism of mesenchymal stem cells for apoptotic cell death. Cell Death Differ. 2015;22(7):1219-1230.

11. Maugeri $\mathrm{N}$, et al. Circulating platelets as a source of the damage-associated molecular pattern HMGB1 in patients with systemic sclerosis. Autoimmunity. 2012;45(8):584-587.

12. Rouhiainen A, Imai S, Rauvala H, Parkkinen J. Occurrence of amphoterin (HMG1) as an endogenous protein of human platelets that is exported to the cell surface upon platelet activation. Thromb Haemost. 2000;84(6):1087-1094.

13. Vogel S, et al. Activated platelets interfere with recruitment of mesenchymal stem cells to apoptotic cardiac cells via high mobility group box $1 /$ Toll-like receptor 4-mediated down-regulation of hepatocyte growth factor receptor MET. J Biol Chem. 2014;289(16):11068-11082.

14. Goldstein RS, et al. Elevated high-mobility group box 1 levels in patients with cerebral and myocardial ischemia. Shock. 2006;25(6):571-574.

15. Kohno $\mathrm{T}$, et al. Role of high-mobility group box 1 protein in post-infarction healing process and left ventricular remodelling. Cardiovasc Res. 2009;81(3):565-573.

16. Wang $\mathrm{H}$, et al. HMG-1 as a late mediator of endotoxin lethality in mice. Science. 1999;285(5425):248-251.

17. Hatada T, et al. Plasma concentrations and importance of High Mobility Group Box protein in the prognosis of organ failure in patients with disseminated intravascular coagulation. Thromb Haemost. 2005;94(5):975-979.

18. Levy RM, et al. Systemic inflammation and remote organ injury following trauma require HMGB1. Am J Physiol Regul Integr Comp Physiol. 2007;293(4):R1538-R1544.

19. Venereau E, Schiraldi M, Uguccioni M, Bianchi
ME. HMGB1 and leukocyte migration during trauma and sterile inflammation. Mol Immunol. 2013;55(1):76-82.

20. Fan J, et al. Hemorrhagic shock induces $\mathrm{NAD}(\mathrm{P}) \mathrm{H}$ oxidase activation in neutrophils: role of HMGB1-TLR4 signaling. J Immunol. 2007;178(10):6573-6580.

21. Huang $\mathrm{H}$, et al. Damage-associated molecular pattern-activated neutrophil extracellular trap exacerbates sterile inflammatory liver injury. Hepatology. 2015;62(2):600-614.

22. Maugeri N, et al. Activated platelets present high mobility group box 1 to neutrophils, inducing autophagy promoting the extrusion of neutrophil extracellular traps. J Thromb Haemost. 2014;12(12):2074-2088.

23. Ito $T$, et al. High-mobility group box 1 protein promotes development of microvascular thrombosis in rats. J Thromb Haemost. 2007;5(1):109-116.

24. Park JS, et al. High mobility group box 1 protein interacts with multiple Toll-like receptors. Am J Physiol Cell Physiol. 2006;290(3):C917-C924.

25. Park JS, et al. Involvement of toll-like receptors 2 and 4 in cellular activation by high mobility group box 1 protein. J Biol Chem. 2004;279(9):7370-7377.

26. Aslam R, et al. Platelet Toll-like receptor expression modulates lipopolysaccharideinduced thrombocytopenia and tumor necrosis factor-alpha production in vivo. Blood. 2006;107(2):637-641.

27. Fuentes E, Rojas A, Palomo I. Role of multiligand/RAGE axis in platelet activation. Thromb Res. 2014;133(3):308-314.

28. Stark RJ, Aghakasiri N, Rumbaut RE. Plateletderived Toll-like receptor 4 (Tlr-4) is sufficien to promote microvascular thrombosis in endotoxemia. PLoS One. 2012;7(7):e41254.

29. Zhang G, et al. Lipopolysaccharide stimulates platelet secretion and potentiates platelet aggregation via TLR4/MyD88 and the cGMP-dependent protein kinase pathway. J Immunol. 2009;182(12):7997-8004.

30. Ding N, et al. Toll-like receptor 4 regulates platelet function and contributes to coagulation abnormality and organ injury in hemorrhagic shock and resuscitation. Circ Cardiovasc Genet. 2014;7(5):615-624.

31. Li Z, et al. A stimulatory role for cGMP-dependent protein kinase in platelet activation. Cell. 2003;112(1):77-86.

32. Li Z, Zhang G, Marjanovic JA, Ruan C, Du $\mathrm{X}$. A platelet secretion pathway mediated by cGMP-dependent protein kinase. J Biol Chem. 2004;279(41):42469-42475.

33. Abrams ST, et al. Circulating histones are mediators of trauma-associated lung injury. Am J Respir Crit Care Med. 2013;187(2):160-169.

34. Gando S. Disseminated intravascular coagulation in trauma patients. Semin Thromb Hemost. 2001;27(6):585-592.

35. Kang R, et al. Intracellular Hmgb1 inhibits inflammatory nucleosome release and limits acute pancreatitis in mice. Gastroenterology. 2014;146(4):1097-1107.

36. Tiedt R, Schomber T, Hao-Shen H, Skoda RC. Pf4-Cre transgenic mice allow the generation of lineage-restricted gene knockouts for studying megakaryocyte and platelet function in vivo. Blood. 2007;109(4):1503-1506.

37. Li Z, Xi X, Du X. A mitogen-activated protein kinase-dependent signaling pathway in the activation of platelet integrin $\alpha I I b \beta 3$. J Biol Chem . 2001;276(45):42226-42232.

38. Aszodi A, et al. The vasodilator-stimulated phosphoprotein (VASP) is involved in cGMPand cAMP-mediated inhibition of agonistinduced platelet aggregation, but is dispensable for smooth muscle function. ЕMBO J. 1999;18(1):37-48.

39. Dostmann WR, Taylor MS, Nickl CK, Brayden JE, Frank R, Tegge WJ. Highly specific, membrane-permeant peptide blockers of cGMPdependent protein kinase I $\alpha$ inhibit NO-induced cerebral dilation. Proc Natl Acad Sci U S A. 2000;97(26):14772-14777.

40. Andersson U, Tracey KJ. HMGB1 is a therapeutic target for sterile inflammation and infection. Anпu Rev Immunol. 2011;29:139-162.

41. Nomura S, et al. The correlation between platelet activation markers and HMGB1 in patients with disseminated intravascular coagulation and hematologic malignancy. Platelets. 2011;22(5):396-397.

42. Ahrens I, et al. HMGB1 binds to activated platelets via the receptor for advanced glycation end products and is present in platelet rich human coronary artery thrombi. Thromb Haemost. 2015;114(5):994-1003.

43. Elvers $\mathrm{M}$, et al. Intracellular cyclophilin $\mathrm{A}$ is an important $\mathrm{Ca}(2+)$ regulator in platelets and critically involved in arterial thrombus formation. Blood. 2012;120(6):1317-1326.

44. Seizer P, et al. Extracellular cyclophilin A activates platelets via EMMPRIN (CD147) and PI3K/Akt signaling, which promotes platelet adhesion and thrombus formation in vitro and in vivo. Arterioscler Thromb Vasc Biol. 2014;35(3):655-663.

45. Boudreau LH, et al. Platelets release mitochondria serving as substrate for bactericidal group IIA-secreted phospholipase A2 to promote inflammation. Blood. 2014;124(14):2173-2183.

46. Chatterjee M, et al. Macrophage migration inhibitory factor limits activation-induced apoptosis of platelets via CXCR7-dependent Akt signaling. Circ Res. 2014;115(11):939-949.

47. Vogel S, Rath D, Lu J, Chatterjee M, Geisler T, Gawaz M. Elevated mitochondrial membrane potential of circulating monocyte-platelet aggregates in patients with coronary heart disease. Int J Cardiol. 2014;181:135-137.

48. Neal MD, et al. Prehospital use of nonsteroidal anti-inflammatory drugs (NSAIDs) is associated with a reduced incidence of trauma-induced coagulopathy. Ann Surg. 2014;260(2):378-382.

49. Gill R, et al. Systemic inflammation and liver injury following hemorrhagic shock and peripheral tissue trauma involve functional TLR9 signaling on bone marrow-derived cells and parenchymal cells. Shock. 2011;35(2):164-170.

50. Caudrillier A, et al. Platelets induce neutrophil extracellular traps in transfusion-related acute lung injury. JClin Invest. 2012;122(7):2661-2671.

51. Fuchs TA, et al. Extracellular DNA traps promote thrombosis. Proc Natl Acad Sci US A. 
2010;107(36):15880-15885.

52. Huang $\mathrm{H}$, et al. Hepatocyte-specific high-mobility group box 1 deletion worsens the injury in liver ischemia/reperfusion: a role for intracellular high-mobility group box 1 in cellular protection. Hepatology. 2014;59(5):1984-1997.

53. Yanai $\mathrm{H}$, et al. Conditional ablation of HMGB1 in mice reveals its protective function against endotoxemia and bacterial infection. Proc Natl Acad Sci U S A. 2013;110(51):20699-20704.

54. Zhu X, et al. Cytosolic HMGB1 controls the cellular autophagy/apoptosis checkpoint during inflammation. JClin Invest. 2015;125(3):1098-1110.

55 . Andrassy M, et al. High-mobility group box-1 in ischemia-reperfusion injury of the heart. Circulation. 2008;117(25):3216-3226.

56. Tsung A, et al. The nuclear factor HMGB1 mediates hepatic injury after murine liver ischemia-reperfusion. JExp Med. 2005;201(7):1135-1143.

57. Rheinlaender J, et al. Imaging the elastic modulus of human platelets during thrombin-induced activation using scanning ion conductance microscopy.
Thromb Haemost. 2015;113(2):305-311.

58. Massberg S, et al. Increased adhesion and aggregation of platelets lacking cyclic guanosine 3', 5'-monophosphate kinase I. J Exp Med. 1999;189(8):1255-1264.

59. Zhang G, et al. Biphasic roles for soluble guanylyl cyclase (sGC) in platelet activation. Blood. 2011;118(13):3670-3679.

60. Cai C, et al. Benzyl alcohol attenuates acetaminophen-induced acute liver injury in a Toll-like receptor-4-dependent pattern in mice. Hepatology. 2014;60(3):990-1002.

61. Sodhi C, et al. DNA attenuates enterocyte Tolllike receptor 4-mediated intestinal mucosal injury after remote trauma. Am J Physiol Gastrointest Liver Physiol. 2011;300(5):G862-G873.

62. Geisel J, et al. IL-6 and maturation govern TLR2 and TLR4 induced TLR agonist tolerance and cross-tolerance in dendritic cells. JImmunol. 2007;179(9):5811-5818.

63. Yazji I, et al. Endothelial TLR4 activation impairs intestinal microcirculatory perfusion in necrotiz- ing enterocolitis via eNOS-NO-nitrite signaling. Proc Natl Acad Sci U S A. 2013;110(23):9451-9456.

64. Weber S, et al. Rescue of cGMP kinase I knockout mice by smooth muscle specific expression of either isozyme. Circ Res. 2007;101(11):1096-1103.

65. Feil R, Lohmann SM, de Jonge H, Walter U, Hofmann F. Cyclic GMP-dependent protein kinases and the cardiovascular system: insights from genetically modified mice. Circ Res. 2003;93(10):907-916.

66. Choudhri TF, et al. Reduced microvascular thrombosis and improved outcome in acute murine stroke by inhibiting GP IIb/IIIa receptor-mediated platelet aggregation. JClin Invest. 1998;102(7):1301-1310.

67. Darwiche SS, Kobbe P, Pfeifer R, Kohut L, Pape HC, Billiar T. Pseudofracture: an acute peripheral tissue trauma model. J Vis Exp. 2011;(50):2074.

68. Lee D, Fong KP, King MR, Brass LF, Hammer DA. Differential dynamics of platelet contact and spreading. Biophys J. 2012;102(3):472-482. 